\title{
FINITE-DIMENSIONAL HALF-INTEGER WEIGHT MODULES OVER QUEER LIE SUPERALGEBRAS
}

\author{
SHUN-JEN CHENG ${ }^{\dagger}$ AND JAE-HOON KWON ${ }^{\dagger \dagger}$
}

\begin{abstract}
We give a new interpretation of representation theory of the finitedimensional half-integer weight modules over the queer Lie superalgebra $\mathfrak{q}(n)$. It is given in terms of Brundan's work of finite-dimensional integer weight $\mathfrak{q}(n)$-modules by means of Lusztig's canonical basis. Using this viewpoint we compute the characters of the finite-dimensional half-integer weight irreducible modules. For a large class of irreducible modules whose highest weights are of special types (i.e., totally connected or totally disconnected) we derive closed-form character formulas that are reminiscent of Kac-Wakimoto character formula for classical Lie superalgebras.
\end{abstract}

\section{INTRODUCTION}

The character problem for finite-dimensional irreducible modules over the basic classical Lie superalgebras has now been settled (see, e.g., Br1, CLW, CL, Ger, GS, Mar, Ser, [Sv1, SZ1, SZ2, vdJ]). For the queer Lie superalgebra $\mathfrak{q}(n)$, the solution of this problem was first given by Penkov and Serganova [PS1, PS2]. Indeed, given dominant weights $\lambda, \mu$ for $\mathfrak{q}(n)$, they provide an algorithm for computing the multiplicity of an irreducible $\mathfrak{q}(n)$-module $L(\mu)$ with highest weight $\mu$ in the cohomology groups of certain bundle on $\Pi$-symmetric projective space associated to $\lambda$, and, as a consequence, they determine the coefficient $a_{\lambda \mu}$ of the character of $L(\mu)$ in the expansion of the character of the associated Euler characteristic $E(\lambda)$.

A completely different approach to computing the transition matrix $\left(a_{\lambda \mu}\right)$ between the characters of $E(\lambda)$ and $L(\mu)$ in the case when $\lambda, \mu$ are integer dominant weights was given by Brundan [Br2]. Indeed, let $U_{q}\left(\mathfrak{b}_{\infty}\right)$ be the quantum group of type $B$ with infinite rank, and let $\mathscr{F}^{n}$ the $n$th exterior power of its natural representation $\mathscr{V}$ (cf. [JMO]). It was shown that $\left(a_{\lambda \mu}\right)$ is given by the transpose of the transition matrix between the canonical basis and the natural monomial basis of $\mathscr{F}^{n}$ at $q=1$. This, together with an explicit combinatorial algorithm for computing the canonical basis elements, gives a solution of the irreducible character problem in the case of integer weight modules. In the equivalent dual picture the Grothendieck group of the category of finite-dimensional integer weight modules can be realized by a $U_{q}\left(\mathfrak{b}_{\infty}\right)$-module $\mathscr{E}^{n}$, which is dual to $\mathscr{F}^{n}$ with respect to a bilinear form on $\mathscr{V}^{\otimes n}$ relating the canonical

2010 Mathematics Subject Classification. 17B67.

${ }^{\dagger}$ Partially supported by a MOST and an Academia Sinica Investigator grant.

${ }^{\dagger \dagger}$ Partially supported by a NRF-grant 2011-0006735. 
and dual canonical basis on the ambient space. In this dual picture $E(\lambda)$ is mapped to a standard basis element $E_{\lambda}$, which in turn is dual to the standard monomial basis of $\mathscr{F}^{n}$, and $L(\mu)$ is mapped to a dual canonical basis element $L_{\lambda}$ at $q=1$. Further discussion and description for the inverse of $\left(a_{\lambda \mu}\right)$ are given in [SZ3]

In this paper we consider the finite-dimensional half-integer weight irreducible $\mathfrak{q}(n)$ modules and their characters. We show that they also can be explained nicely in terms of the Brundan's results on integer weight modules. To be more precise, we take a quotient space $\mathscr{E}^{n, \times}$ of $\mathscr{E}^{n}$ by a subspace spanned by standard basis elements $E_{\lambda}$, where $\lambda$ has at least one zero part considered as a generalized partition. Then we show that the transition matrix $\left(a_{\lambda \mu}\right)$ for half-integer dominant weights $\lambda$ and $\mu$ is the same as the transition matrix $\left(a_{\lambda^{\natural} \mu^{\natural}}\right)$ between the two bases of $\mathscr{E} n, \times$ given by $\left\{\mathbf{E}_{\lambda^{\natural}}\right\}$ and $\left\{\mathbf{L}_{\mu^{\natural}}\right\}$ respectively (Theorem 6.4), where $\downarrow$ is a natural bijection from the set of halfinteger dominant weights to the set of integer dominant weights with no zero parts (see (2.2) ). Here, the notations $\mathbf{E}_{\lambda^{\natural}}$ and $\mathbf{L}_{\mu^{\natural}}$ stand for the images of $E_{\lambda^{\natural}}$ and $L_{\mu^{\natural}}$ under the canonical projection from $\mathscr{E}^{n}$ onto $\mathscr{E}^{n, \times}$. We remark that $\mathscr{E}^{n, \times}$ also has a well-defined bar involution induced from that of $\mathscr{E}^{n}$, and $\left\{\mathbf{L}_{\mu^{\natural}}\right\}$ forms a unique bar-invariant basis of $\mathscr{E}^{n}, \times$ satisfying the properties of the dual canonical basis, although $\mathscr{E}^{n, \times}$ is certainly not a $U_{q}\left(\mathfrak{b}_{\infty}\right)$-module.

In particular, we have (up to some shift of weights) the same combinatorial formula for $a_{\lambda \mu}$ for half-integer dominant weights as in [Br2]. Furthermore, we obtain a nice closed-form formula for the inverse matrix of $\left(a_{\lambda \mu}\right)$ and hence for the character formula for $L(\lambda)$ as well (Theorem 6.6), which turns out to be essentially the same as the formula for general linear Lie superalgebras given in Br1]. This is proved by establishing a correspondence from half-integer dominant weights for $\mathfrak{q}(n)$ to integer dominant weights for for the general linear Lie superalgebra $\mathfrak{g l}(p \mid q)$ for some $p, q$ with $p+q=n$ preserving the Bruhat orders on each weight lattice. As an interesting application, we obtain KacWakimoto type character formulas for $L(\lambda)$ when $\lambda$ is either a totally connected or a totally disconnected half-integer dominant weight (Theorem 7.1).

We conclude this introduction with the organization of the paper. In Section 2, we briefly recall some preliminary background and notations. In Section 3, we compare the Bruhat order in PS2] with the ones in [Br1, Br2]. In Section 4, we present the Euler characteristic in a purely algebraic way using the dual Zuckerman functor and establish some of its basic properties. In Section 5, we prove our main observation on $a_{\lambda \mu}$ for half-integer dominant weights by analyzing the algorithm of Penkov and Serganova. In Section 6, we realize the Grothendieck group for the finite-dimensional half-integer weight modules, and compute the irreducible characters. Finally, as an application, Kac-Wakimoto type character formulas [KW are given in Section 7 for finite-dimensional irreducible half-integer modules whose highest weights are either totally connected or totally disconnected. Note that, just as in the case of classical Lie superalgebras, our formulas can then be regarded as generalizations of the BernsteinLeites character formula $\mathrm{BL}$. 
Acknowledgment. We thank Dimitar Grantcharov for raising with the first author the question about possible Kac-Wakimoto type character formula for the queer Lie superalgebra, which was one of the original motivation for investigation in this paper. The second author thanks Academia Sinica in Taipei for hospitality and support, where part of this work was carried out.

Notation. Let $\mathbb{Z}, \mathbb{N}$, and $\mathbb{Z}_{+}$stand for the sets of all, positive, and non-negative integers, respectively. All vector spaces, algebras, etc., are over the complex field $\mathbb{C}$.

\section{Preliminaries}

Fix a positive integer $n \geq 1$. Let $\mathbb{C}^{n \mid n}$ be the complex superspace of dimension $(n \mid n)$. Choose an ordered basis $\left\{v_{\overline{1}}, \ldots, v_{\bar{n}}\right\}$ for the even subspace $\mathbb{C}^{n \mid 0}$ and an ordered basis $\left\{v_{1}, \ldots, v_{n}\right\}$ for the odd subspace $\mathbb{C}^{0 \mid n}$ so that the general linear Lie superalgebra $\mathfrak{g l}(n \mid n)$ may be realized as $2 n \times 2 n$ complex matrices indexed by $I(n \mid n):=\{\overline{1}<\ldots<$ $\bar{n}<1<\ldots<n\}$. The subspace

$$
\mathfrak{q}(n):=\left\{\left(\begin{array}{ll}
A & B \\
B & A
\end{array}\right) \mid A, B: n \times n \text { matrices }\right\}
$$

forms a subalgebra of $\mathfrak{g l}(n \mid n)$ called the queer Lie superalgebra.

Let $\mathfrak{g}$ stand for the Lie superalgebra $\mathfrak{q}(n)$ from now on. We denote by $E_{a b}$ the elementary matrix in $\mathfrak{g l}(n \mid n)$ with $(a, b)$-entry 1 and other entries 0 , for $a, b \in I(n \mid n)$. We have a linear basis $\left\{e_{i j}, \bar{e}_{i j} \mid 1 \leq i, j \leq n\right\}$ of $\mathfrak{g}$, where $e_{i j}=E_{\overline{i j}}+E_{i j}$ and $\bar{e}_{i j}=$ $E_{\bar{i} j}+E_{i \bar{j}}$. Note that $\mathfrak{g}_{\overline{0}}$ is spanned by $\left\{e_{i j} \mid 1 \leq i, j \leq n\right\}$, which is isomorphic to the general linear Lie algebra $\mathfrak{g l}(n)$.

Let $\mathfrak{h}=\mathfrak{h}_{\overline{0}} \oplus \mathfrak{h}_{\overline{1}}$ be the standard Cartan subalgebra of $\mathfrak{g}$, which consists of matrices of the form (2.1) with $A$ and $B$ diagonal. Then $\left\{h_{i}:=e_{i i} \mid 1 \leq i \leq n\right\}$ and $\left\{\bar{h}_{i}:=\right.$ $\left.\bar{e}_{i i} \mid 1 \leq i \leq n\right\}$ form linear bases of $\mathfrak{h}_{\overline{0}}$ and $\mathfrak{h}_{\overline{1}}$, respectively. Let $\left\{\varepsilon_{i} \mid 1 \leq i \leq n\right\}$ be the basis of $\mathfrak{h}_{\overline{0}}^{*}$ dual to $\left\{h_{i} \mid 1 \leq i \leq n\right\}$. We define a symmetric bilinear form $($, on $\mathfrak{h}_{\overline{0}}^{*}$ by $\left(\varepsilon_{i}, \varepsilon_{j}\right)=\delta_{i j}$, for $1 \leq i, j \leq n$. Let $\mathfrak{b}$ be the standard Borel subalgebra of $\mathfrak{g}$, which consists of matrices of the form (2.1) with $A$ and $B$ upper triangular. We have $\mathfrak{b}=\mathfrak{h} \oplus \mathfrak{n}$, where $\mathfrak{n}$ is the nilradical spanned by $\left\{e_{i j}, \bar{e}_{i j} \mid 1 \leq i<j \leq n\right\}$. Also, denote by $\mathfrak{n}_{-}$the opposite nilradical so that $\mathfrak{g}=\mathfrak{b} \oplus \mathfrak{n}_{-}$. We denote by $\Phi^{+}$and $\Phi^{-}$the sets of positive and negative roots with respect to $\mathfrak{h}_{\overline{0}}$, respectively, and denote by $\Pi$ the set of simple roots of $\mathfrak{g}_{0}$. We have $\Phi^{-}=-\Phi^{+}, \Pi=\left\{\varepsilon_{i}-\varepsilon_{i+1} \mid 1 \leq i \leq n-1\right\}$, and $\Phi^{+}=\Phi_{\overline{0}}^{+} \sqcup \Phi_{\overline{1}}^{+}$, where $\Phi_{\overline{0}}^{+}$and $\Phi_{\overline{1}}^{+}$stand for the sets of positive even and odd roots, respectively. Ignoring parity we have $\Phi_{\overline{0}}^{+}=\Phi_{\overline{1}}^{+}=\left\{\varepsilon_{i}-\varepsilon_{j} \mid 1 \leq i<j \leq n\right\}$.

Furthermore, we denote by $W$, the Weyl group of $\mathfrak{g}$, which is the Weyl group of the reductive Lie algebra $\mathfrak{g}_{\overline{0}}$ and hence acts naturally on $\mathfrak{h}_{\overline{0}}^{*}$ by permutation. We also denote by $\ell(w)$ the length of an element $w \in W$. 
For a $\mathfrak{g}$-module $V$ and $\mu \in \mathfrak{h}_{\overline{0}}^{*}$, let $V_{\mu}=\left\{v \in V \mid h \cdot v=\mu(h) v\right.$ for $\left.h \in \mathfrak{h}_{\overline{0}}\right\}$ denote its $\mu$-weight space. For a $\mathfrak{g}$-module $V$ with weight space decomposition $V=\bigoplus_{\mu \in \mathfrak{h}_{\overline{0}}^{*}} V_{\mu}$, its character is defined by $\operatorname{ch} V:=\sum_{\mu \in \mathfrak{h}_{0}^{*}} \operatorname{dim} V_{\mu} e^{\mu}$, where $e$ is an indeterminate.

Let $\lambda=\sum_{i=1}^{n} \lambda_{i} \varepsilon_{i} \in \mathfrak{h}_{0}^{*}$ be given. We put $\ell(\lambda)$ to be the number of $i$ 's with $\lambda_{i} \neq 0$. Consider a symmetric bilinear form on $\mathfrak{h}_{\overline{1}}$ given by $\langle\cdot, \cdot\rangle_{\lambda}=\lambda([\cdot, \cdot])$, and let $\mathfrak{h}_{\overline{1}}^{\prime}$ be a maximal isotropic subspace associated to $\langle\cdot, \cdot\rangle_{\lambda}$. Put $\mathfrak{h}^{\prime}=\mathfrak{h}_{\overline{0}} \oplus \mathfrak{h}_{\overline{1}}^{\prime}$. Let $\mathbb{C} v_{\lambda}$ be the onedimensional $\mathfrak{h}^{\prime}$-module with $h \cdot v_{\lambda}=\lambda(h) v_{\lambda}$ and $h^{\prime} \cdot v_{\lambda}=0$, for $h \in \mathfrak{h}_{\overline{0}}$ and $h^{\prime} \in \mathfrak{h}_{\overline{1}}^{\prime}$. Then $W_{\lambda}:=\operatorname{Ind}_{\mathfrak{h}^{\prime}}^{\mathfrak{h}} \mathbb{C} v_{\lambda}$ is an irreducible $\mathfrak{h}$-module of dimension $2^{\lceil\ell(\lambda) / 2\rceil}$, where $\lceil\cdot\rceil$ denotes the ceiling function. We put $\Delta(\lambda)=\operatorname{Ind}_{\mathfrak{b}}^{\mathfrak{g}} W_{\lambda}$, where $W_{\lambda}$ is extended to a $\mathfrak{b}$-module in a trivial way, and define $L(\lambda)$ to be the unique irreducible quotient of $\Delta(\lambda)$. Note that it is an $\mathfrak{h}_{\overline{0}}$-semisimple highest weight $\mathfrak{g}$-module of highest weight $\lambda$. The center of $\mathfrak{g}$ was determined by Sergeev [Sv2]. We denote by $\chi_{\lambda}$ the central character of $L(\lambda)$ (see, e.g., [CW, Section 2.3] for more details). We say that a positive root $\varepsilon_{i}-\varepsilon_{j}(i<j)$ is atypical to $\lambda$ if $\lambda_{i}+\lambda_{j}=0$. Recall that a weight $\lambda$ is said to be atypical if there is a positive root atypical to $\lambda$, and typical otherwise $[\mathrm{Pe}$. The degree of atypicality of $\lambda$ is the maximal number of positive even roots which are mutually orthogonal and atypical to $\lambda$.

For $\epsilon=0, \frac{1}{2}$, let $\Lambda_{\epsilon+\mathbb{Z}}:=\sum_{i=1}^{n}(\epsilon+\mathbb{Z}) \varepsilon_{i} \subseteq \mathfrak{h}_{0}^{*}$ and put $\Lambda=\Lambda_{\mathbb{Z}} \cup \Lambda_{\frac{1}{2}+\mathbb{Z}}$. We say that a $\mathfrak{g}$-module $V$ is an integer weight module and half-integer weight module if it has a weight space decomposition $V=\bigoplus_{\mu \in \Lambda_{\epsilon+\mathbb{Z}}} V_{\mu}$ for $\epsilon=0$ and $\frac{1}{2}$, respectively.

Let $\mathcal{O}_{\mathbb{Z}}$ and $\mathcal{O}_{\frac{1}{2}+\mathbb{Z}}$ denote the BGG categories of $\mathfrak{h}_{\overline{0}}$-semisimple integer weight and half-integer weight $\mathfrak{g}$-modules, respectively. Let $\mathcal{O}^{\text {fin }}$ be the category of finite-dimensional $\mathfrak{g}$-modules. Set

$$
\mathcal{O}:=\mathcal{O}_{\mathbb{Z}} \cup \mathcal{O}_{\frac{1}{2}+\mathbb{Z}}, \quad \mathcal{O}_{\epsilon+\mathbb{Z}}^{\text {fin }}:=\mathcal{O}_{\epsilon+\mathbb{Z}} \cap \mathcal{O}^{\text {fin }}
$$

for $\epsilon=0, \frac{1}{2}$. For a module category $\mathcal{C}$ given above, we denote by $K(\mathcal{C})$ the corresponding Grothendieck group spanned by $[M](M \in \mathcal{C})$, where $[M]$ stands for the equivalence class of the module $M$.

Define

$$
\Lambda_{\epsilon+\mathbb{Z}}^{+}:=\left\{\lambda=\sum_{i=1}^{n} \lambda_{i} \varepsilon_{i} \in \Lambda_{\epsilon+\mathbb{Z}} \mid \lambda_{i} \geq \lambda_{i+1}, \lambda_{i}=\lambda_{i+1} \text { implies } \lambda_{i}=0 \text { for all } i\right\},
$$

for $\epsilon=0, \frac{1}{2}$, and put $\Lambda^{+}=\Lambda_{\mathbb{Z}}^{+} \cup \Lambda_{\frac{1}{2}+\mathbb{Z}}^{+}$. We call a weight $\nu$ in $\Lambda^{+}, \Lambda_{\mathbb{Z}}^{+}$and $\Lambda_{\frac{1}{2}+\mathbb{Z}}^{+}$ dominant, integer dominant, and half-integer dominant, respectively. According to [Pe, Theorem 4] we have $L(\lambda) \in \mathcal{O}_{\epsilon+\mathbb{Z}}^{\text {fin }}$ if and only if $\lambda \in \Lambda_{\epsilon+\mathbb{Z}}^{+}$(see also [CW], Theorem 2.18]). We also let

$$
\Lambda_{\mathbb{Z}^{\times}}:=\left\{\lambda \in \Lambda_{\mathbb{Z}} \mid \lambda_{i} \neq 0 \text { for all } i\right\}, \quad \Lambda_{\mathbb{Z}^{\times}}^{+}:=\Lambda^{+} \cap \Lambda_{\mathbb{Z}^{\times}},
$$


and let a bijection $\sharp: \Lambda_{\frac{1}{2}+\mathbb{Z}} \rightarrow \Lambda_{\mathbb{Z} \times}$ be given by

$$
\lambda=\sum_{i=1}^{n} \lambda_{i} \varepsilon_{i} \longmapsto \lambda^{\sharp}:=\sum_{i=1}^{n}\left(\lambda_{i}+\operatorname{sgn}\left(\lambda_{i}\right) \frac{1}{2}\right) \varepsilon_{i} .
$$

\section{BRUHAT ORDERINGS}

Let $\Lambda^{\geqslant}=\left\{\lambda=\sum_{i=1}^{n} \lambda_{i} \varepsilon_{i} \in \Lambda \mid \lambda_{i} \geq \lambda_{i+1}\right.$ for all $\left.i\right\}$ be the set of $\mathfrak{g}_{0_{0}}$-dominant weights. We have $\Lambda^{+} \subset \Lambda^{\geqslant}$. Put $\Lambda_{\epsilon+\mathbb{Z}}^{\geqslant}=\Lambda^{\geqslant} \cap \Lambda_{\epsilon+\mathbb{Z}}$ for $\epsilon=0, \frac{1}{2}$, and $\Lambda_{\mathbb{Z}^{\times}}^{\geqslant}=\Lambda^{\geqslant} \cap \Lambda_{\mathbb{Z}^{\times}}$. For $\lambda, \mu \in \Lambda^{\geqslant}$, we define the Bruhat ordering $\succcurlyeq$ as in [PS2, Lemma 2.1]: $\lambda \succcurlyeq \mu$ if and only if there exists a sequence of elements $\mu=\nu_{(1)}, \ldots, \nu_{(k)}=\lambda$ in $\Lambda^{\geqslant}$and roots $\beta_{i} \in \Phi^{+}$such that $\nu_{(i)}+\beta_{i}=\nu_{(i+1)}$ with $\left(\nu_{(i)}, \beta_{i}\right)=0$ for $1 \leq i \leq k-1$.

Let us say that a subset $A$ of a partially ordered set $(S, \geq)$ is increasing if we have $s \in A$ for any $a \in A$ and $s \in S$ with $s \geq a$.

Lemma 3.1. The set $\Lambda_{\mathbb{Z}^{\times}}^{\geqslant}$is an increasing subset of $\left(\Lambda^{\geqslant}, \succcurlyeq\right)$.

Proof. Let $\lambda, \mu \in \Lambda^{\geqslant}$such that $\lambda \succcurlyeq \mu$. We will show that if $\mu \in \Lambda_{\mathbb{Z}^{\times}}^{\geqslant}$, then $\lambda \in \Lambda_{\mathbb{Z}^{\times}}^{\geqslant}$. It is enough to consider the case $\lambda-\mu=\varepsilon_{i}-\varepsilon_{j}$ with $i<j$ and $\left(\mu, \varepsilon_{i}-\varepsilon_{j}\right)=0$. In this case we have $\mu_{i}>0$ and $\mu_{j}=-\mu_{i}<0$. Thus $\lambda_{i}=\mu_{i}+1>0$ and $\lambda_{j}=\mu_{j}-1<0$. As $\lambda_{s}=\mu_{s}$, for all $s \neq i, j$, the lemma follows.

Lemma 3.2. For $\lambda, \mu \in \Lambda_{\frac{1}{2}+\mathbb{Z}}^{\geqslant}$, we have $\lambda \succcurlyeq \mu$ if and only if $\lambda^{\sharp} \succcurlyeq \mu^{\sharp}$.

Proof. Suppose that $\lambda \succcurlyeq \mu$. Again it is enough to prove the case when $\lambda-\mu=\varepsilon_{i}-\varepsilon_{j}$ and $\left(\mu, \varepsilon_{i}-\varepsilon_{j}\right)=0$ for some $1 \leq i<j \leq n$. We have $\lambda_{i}=\mu_{i}+1>\mu_{i}>0>\mu_{j}>\mu_{j}-1=\lambda_{j}$ with $\mu_{i}+\mu_{j}=0$. Then it is clear that $\lambda^{\sharp}-\mu^{\sharp}=\varepsilon_{i}-\varepsilon_{j}$ and hence $\lambda^{\sharp} \succcurlyeq \mu^{\sharp}$.

Conversely, suppose that $\lambda^{\sharp} \succcurlyeq \mu^{\sharp}$. By Lemma 3.1 we have $\eta \in \Lambda_{\mathbb{Z}^{\times}}^{\geqslant}$for any $\eta \in \Lambda_{\mathbb{Z}}^{\geqslant}$ such that $\lambda^{\sharp} \succcurlyeq \eta \succcurlyeq \mu^{\sharp}$, that is, $\eta=\nu^{\sharp}$ for some (unique) $\nu \in \Lambda_{\frac{1}{2}+\mathbb{Z}}^{\geqslant}$. Then it is clear that $\lambda \succcurlyeq \mu$ by the same argument as in the above paragraph.

In [Br2, Section 2.3] a partial ordering $\succeq$ on $\Lambda_{\mathbb{Z}}$ was defined based on the root lattice of the Lie algebra of type $\mathfrak{b}_{\infty}$ (i.e., type $B$ of infinite rank), which we shall recall below. We will show that this ordering on $\Lambda_{\mathbb{Z}}^{\geqslant}$is equivalent to $\succcurlyeq$ in Proposition 3.3 below. So there will be no confusion to call both orderings Bruhat ordering in this paper.

Let $\mathrm{P}$ be the free abelian group with orthonormal basis $\left\{\delta_{r} \mid r \in \mathbb{N}\right\}$ with respect to a bilinear form $(\cdot, \cdot)$. We define a partial ordering on $\mathrm{P}$ by declaring $\nu \geq \eta$, for $\nu, \eta \in \mathrm{P}$, if $\nu-\eta$ is a non-negative integral linear combination of the positive simple roots of type $\mathfrak{b}_{\infty}$, that is, $-\delta_{1}$ and $\delta_{r}-\delta_{r+1}(r \in \mathbb{N})$.

For $\lambda=\sum_{i=1}^{n} \lambda_{i} \varepsilon_{i} \in \Lambda_{\mathbb{Z}}$, let

$$
\mathrm{wt}_{s}(\lambda):=\sum_{s \leq i \leq n} \delta_{\lambda_{i}} \in \mathrm{P}, \quad \operatorname{wt}(\lambda):=\mathrm{wt}_{1}(\lambda) \in \mathrm{P},
$$


for $1 \leq s \leq n$, where by definition we have $\delta_{-r}=-\delta_{r}$ for $r \in \mathbb{N}$ and $\delta_{0}=0$. Then we define a partial order $\succeq$ on $\Lambda_{\mathbb{Z}}$ as follows: $\lambda \succeq \mu$ if and only if $\operatorname{wt}(\lambda)=\operatorname{wt}(\mu)$ and $\mathrm{wt}_{s}(\lambda) \geq \mathrm{wt}_{s}(\mu)$ for all $1 \leq s \leq n$.

Proposition 3.3. For $\lambda, \mu \in \Lambda_{\mathbb{Z}}^{\geqslant}$, we have $\lambda \succcurlyeq \mu$ if and only if $\lambda \succeq \mu$.

Proof. Let $\lambda=\sum_{i=1}^{n} \lambda_{i} \varepsilon_{i}$ and $\mu=\sum_{i=1}^{n} \mu_{i} \varepsilon_{i}$. Suppose that $\lambda \succcurlyeq \mu$. If is enough to show the case when $\lambda-\mu=\varepsilon_{i}-\varepsilon_{j}$ with $i<j$ and $\left(\mu, \varepsilon_{i}-\varepsilon_{j}\right)=0$. Let $a=\lambda_{i}$. Then

$$
\mathrm{wt}_{s}(\lambda)-\mathrm{wt}_{s}(\mu)= \begin{cases}0, & \text { if } j<s \leq n, \\ \delta_{a-1}-\delta_{a}, & \text { if } i<s \leq j \text { and } a>1, \\ -\delta_{1}, & \text { if } i<s \leq j \text { and } a=1, \\ 0, & \text { if } 1 \leq s \leq i,\end{cases}
$$

which implies that $\lambda \succeq \mu$.

Next, suppose that $\lambda \succeq \mu$. Choose a $p$ such that $\lambda_{i}, \mu_{i} \geq 0$ for $1 \leq i \leq p$, and $\lambda_{j}, \mu_{j} \leq 0$ for $p+1 \leq j \leq n$. Let us identify $\lambda, \mu$ with sequences

$$
\left(a_{1}, \ldots, a_{p} \mid b_{1}, \ldots, b_{q}\right), \quad\left(a_{1}^{\prime}, \ldots, a_{p}^{\prime} \mid b_{1}^{\prime}, \ldots, b_{q}^{\prime}\right),
$$

respectively, where $a_{i}=\lambda_{i}, a_{i}^{\prime}=\mu_{i}$ for $1 \leq i \leq p$, and $b_{j}=-\lambda_{p+j}, b_{j}^{\prime}=-\mu_{p+j}$ for $1 \leq j \leq q:=n-p$. Let $K(\lambda, \mu)=A-A^{\prime}+B-B^{\prime}$, where $A=\sum_{i} a_{i}, A^{\prime}=\sum_{i} a_{i}^{\prime}$, $B=\sum_{j} b_{j}$, and $B^{\prime}=\sum_{j} b_{j}^{\prime}$.

We see first that $A \geq A^{\prime}$ by [Br2, Lemma 2.15]. We claim next that $b_{j} \geq b_{j}^{\prime}$ for all $j$. It is clear that $b_{q} \geq b_{q}^{\prime}$ since $\mathrm{wt}_{q}(\lambda) \geq \mathrm{wt}_{q}(\mu)$. Suppose that there exists a $k$ such that $b_{j} \geq b_{j}^{\prime}$ for $k<j \leq q$ but $0 \leq b_{k}<b_{k}^{\prime}$. Let $a=b_{k}$ and $a^{\prime}=b_{k}^{\prime}$. Then we have

$$
\mathrm{wt}_{p+k}(\lambda)-\mathrm{wt}_{p+k}(\mu)=\mathrm{wt}_{p+k+1}(\lambda)-\mathrm{wt}_{p+k+1}(\mu)-\left(\delta_{a}-\delta_{a^{\prime}}\right),
$$

which contradicts the fact that $\mathrm{wt}_{p+k}(\lambda) \geq \mathrm{wt}_{p+k}(\mu)$ since $\mathrm{wt}_{p+k+1}(\lambda)-\mathrm{wt}_{p+k+1}(\mu)$ is a non-negative integral combination of $\delta_{r}-\delta_{r+1}$ for $r \geq a^{\prime}$. This proves the claim. In particular, we have $B \geq B^{\prime}$, and hence $K(\lambda, \mu) \geq 0$.

Suppose that $K(\lambda, \mu)=0$, equivalently, $A=A^{\prime}$ and $B=B^{\prime}$. Then we have $b_{j}=b_{j}^{\prime}$ for all $1 \leq j \leq q$ since $b_{j} \geq b_{j}^{\prime}$ for $1 \leq j \leq q$. Suppose that $a_{i} \neq a_{i}^{\prime}$ for some $1 \leq i \leq p$. Suppose that there exists a $k$ such that $a_{i} \leq a_{i}^{\prime}$ for $k<i \leq n$ and $a_{k}>a_{k}^{\prime}$. Then

$$
\mathrm{wt}_{k}(\lambda)-\mathrm{wt}_{k}(\mu)=\mathrm{wt}_{k+1}(\lambda)-\mathrm{wt}_{k+1}(\mu)-\left(\delta_{a^{\prime}}-\delta_{a}\right),
$$

where $a=a_{k}$ and $a^{\prime}=a_{k}^{\prime}$. This yields the same contradiction as in (3.2). So we must have $a_{i}^{\prime} \geq a_{i}$ and hence $a_{i}^{\prime}=a_{i}$ for $1 \leq i \leq n$ since $A=A^{\prime}$.

Now, we use induction on $K(\lambda, \mu)$ and $n$ to show that $\lambda \succcurlyeq \mu$. If $n=1$, then it is clear. Also, if $K(\lambda, \mu)=0$, then we have $\lambda=\mu$ by the argument in the previous paragraph. So we assume that $K(\lambda, \mu)>0$ and $n \geq 2$.

If $q=0$, then it is clear that $\lambda=\mu$. If $q \geq 1$ and $b_{q}=b_{q}^{\prime}$, then we may apply the induction hypothesis to the weights

$$
\left(a_{1}, \ldots, a_{p} \mid b_{1}, \ldots, b_{q-1}\right), \quad\left(a_{1}^{\prime}, \ldots, a_{p}^{\prime} \mid b_{1}^{\prime}, \ldots, b_{q-1}^{\prime}\right),
$$


for $\mathfrak{q}(n-1)$ to conclude that $\lambda \succcurlyeq \mu$.

Suppose that $q \geq 1$ and $b_{q}>b_{q}^{\prime}$. Then $b_{j}^{\prime} \neq b_{q}$ for all $1 \leq j \leq q$, and hence $a_{i}=b_{q}$ for some $1 \leq i \leq p$. Put $a=a_{i}=b_{q}$. Consider

$$
\gamma:=\lambda-\varepsilon_{u}+\varepsilon_{v}=\left(a_{1}, \ldots, a_{u}-1, \ldots, a_{p} \mid b_{1}, \ldots, b_{v}-1, \ldots, b_{q}\right),
$$

where $u$ and $v$ are such that $a_{u}=a>a_{u+1}$ and $b_{v+1}<b_{v}=a$. It is clear that $\lambda \succcurlyeq \gamma$. On the other hand, we have

$$
\mathrm{wt}_{s}(\gamma)-\mathrm{wt}_{s}(\mu)= \begin{cases}\mathrm{wt}_{s}(\lambda)-\mathrm{wt}_{s}(\mu), & \text { if } v<s \leq n, \\ \mathrm{wt}_{s}(\lambda)-\mathrm{wt}_{s}(\mu)-\delta_{a-1}+\delta_{a}, & \text { if } u<s \leq v \text { and } a>1, \\ \mathrm{wt}_{s}(\lambda)-\mathrm{wt}_{s}(\mu)+\delta_{1}, & \text { if } u<s \leq v \text { and } a=1, \\ \mathrm{wt}_{s}(\lambda)-\mathrm{wt}_{s}(\mu), & \text { if } 1 \leq s \leq u .\end{cases}
$$

Assume that when $u<s \leq v$ and $a>1$, we have $\operatorname{wt}_{s}(\lambda)-\operatorname{wt}_{s}(\mu)=c_{s, 0}\left(-\delta_{1}\right)+$ $\sum_{1 \leq r \leq a-2} c_{s, r}\left(\delta_{r}-\delta_{r+1}\right)+c_{s, a-1}\left(\delta_{a-1}-\delta_{a}\right)$ for some $c_{s, r} \in \mathbb{Z}_{+}(0 \leq r \leq a-1)$. Since $b_{j}^{\prime}<b_{q}=a$ for all $j$, we have $c_{s, a-1} \geq q-v+1$, and so

$$
\begin{aligned}
& \mathrm{wt}_{s}(\lambda)-\mathrm{wt}_{s}(\mu)-\delta_{a-1}+\delta_{a} \\
& =c_{s, 0}\left(-\delta_{1}\right)+\sum_{1 \leq r \leq a-2} c_{s, r}\left(\delta_{r}-\delta_{r+1}\right)+\left(c_{s, a-1}-1\right)\left(\delta_{a-1}-\delta_{a}\right),
\end{aligned}
$$

which implies that $\gamma \succeq \mu$. Similarly, when when $u<s \leq v$ and $a=1$, we have $\mathrm{wt}_{s}(\lambda)-\mathrm{wt}_{s}(\mu)=c_{s, 0}\left(-\delta_{1}\right)$ for some $c_{s, 0} \geq 1$, and hence $\mathrm{wt}_{s}(\lambda)-\mathrm{wt}_{s}(\mu)+\delta_{1}=$ $\left(c_{s, 0}-1\right)\left(-\delta_{1}\right)$, which also implies $\gamma \succeq \mu$. Since $K(\gamma, \mu)<K(\lambda, \mu)$, we have $\gamma \succcurlyeq \mu$ by induction hypothesis. Therefore, $\lambda \succcurlyeq \mu$. The proof completes.

Corollary 3.4. For $\lambda, \mu \in \Lambda_{\frac{1}{2}+\mathbb{Z}}^{+}$, we have $\lambda \succcurlyeq \mu$ if and only if $\lambda^{\sharp} \succeq \mu^{\sharp}$.

The Bruhat order $\succeq$ on $\Lambda_{\mathbb{Z}}$ is also related with the Bruhat order on the integral weight lattice for the general linear Lie superalgebra in [Br1, Section 2-b] as follows. For $0 \leq p \leq n$ and $q=n-p$, let $\mathbb{Z}^{p \mid q}$ be the set of integer-valued functions on $J(p \mid q)=\{-p<\cdots<-1<1<\cdots<q\}$. We shall also identify an element $f \in \mathbb{Z}^{p \mid q}$ with the sequence of integers

$$
f=(f(-p), \ldots, f(-1) \mid f(1), \ldots, f(q)),
$$

so that $\mathbb{Z}^{p \mid q}$ can be understood as the integral weight lattice for $\mathfrak{g l}(p \mid q)$. Let $P$ be the free abelian group with orthonormal basis $\left\{\gamma_{r} \mid r \in \mathbb{Z}\right\}$ with respect to a bilinear form $(\cdot, \cdot)$. We define a partial order on $P$ by declaring $\nu \geq \eta$, for $\nu, \eta \in P$, if $\nu-\eta$ is a non-negative integral linear combination of the positive simple roots of $\mathfrak{g l}_{\infty}$, that is, $\gamma_{r}-\gamma_{r+1}(r \in \mathbb{Z})$. For $f \in \mathbb{Z}^{p \mid q}$, let

$$
\mathrm{wt}_{s}(f):=\sum_{s \leq i \leq n} \operatorname{sgn}(i) \gamma_{f(i)} \in P, \quad \operatorname{wt}(f):=\mathrm{wt}_{-p}(f) \in P
$$


for $s \in J(p \mid q)$. Then for $f, g \in \mathbb{Z}^{p \mid q}$ we define $f \succeq_{a} g$ if and only if $\operatorname{wt}(f)=\operatorname{wt}(g)$ and $\mathrm{wt}_{s}(f) \geq \mathrm{wt}_{s}(g)$ for all $s \in J(p \mid q)$.

Now, let

$$
\Lambda_{\mathbb{Z}^{\times}}^{\geqslant}(p):=\left\{\lambda=\sum_{i=1}^{n} \lambda_{i} \varepsilon_{i} \in \Lambda_{\mathbb{Z}^{\times}}^{\geqslant} \mid \lambda_{p}>0>\lambda_{p+1}\right\} .
$$

We have $\Lambda_{\mathbb{Z}^{\times}}^{\geqslant}=\bigsqcup_{p=0}^{n} \Lambda_{\mathbb{Z}^{\times}}^{\geqslant}(p)$. For $\lambda \in \Lambda_{\mathbb{Z}^{\times}}^{\geqslant}(p)$, define

$$
\lambda^{b}:=\left(-\lambda_{1}, \ldots,-\lambda_{p} \mid \lambda_{p+1}, \ldots, \lambda_{n}\right) \in \mathbb{Z}^{p \mid q},
$$

(cf. (3.1) ). Let $\mathbb{Z}^{p \mid q}$ be the set of $f \in \mathbb{Z}^{p \mid q}$ such that $f(-p) \leq \cdots \leq f(-1)$ and $f(1) \geq \cdots \geq f(q)$.

Lemma 3.5. The map $b$ is an injection and the image of $\Lambda_{\mathbb{Z}^{\times}}^{\geqslant}(p)$ under $b$ is an increasing subset of $\left(\mathbb{Z}_{\geqslant}^{p \mid q}, \succeq_{a}\right)$ for $0 \leq p \leq n$.

Proof. It follows directly from [Br1, Lemma 2.5].

Proposition 3.6. For $\lambda, \mu \in \Lambda_{\mathbb{Z}^{x}}^{\geqslant}$, we have $\lambda \succeq \mu$ if and only if $\lambda^{b} \succeq_{a} \mu^{b}$.

Proof. Suppose that $\lambda \succeq \mu$. By Lemma 3.1 and Proposition 3.3. there exists a sequence of elements $\mu=\nu_{(1)}, \ldots, \nu_{(k)}=\lambda$ in $\Lambda_{\mathbb{Z}^{\times}}^{\geqslant}$and roots $\beta_{i} \in \Phi^{+}$such that $\nu_{(i)}+\beta_{i}=\nu_{(i+1)}$ with $\left(\nu_{(i)}, \beta_{i}\right)=0$ for $1 \leq i \leq k-1$. Then we have $\lambda, \mu \in \Lambda_{\mathbb{Z}^{\times}}^{\geqslant}(p)$ for some $p$, and $\nu_{(i+1)}^{b}=\nu_{(i)}^{b}-d_{-u_{i}}-d_{v_{i}}$ for some $1 \leq u_{i} \leq p$ and $1 \leq v_{i} \leq q$, where $d_{s} \in \mathbb{Z}^{p \mid q}$ is given by $d_{s}(t)=\delta_{s t}$ for $s, t \in J(p \mid q)$. (This implies $\nu_{(i+1)}^{b} \downarrow \nu_{(i)}^{b}$ following the notation in [Br1, Section 2-b].) Thus we have $\lambda^{b} \succeq_{a} \mu^{b}$ by [Br1, Lemma 2.5].

Conversely, suppose that $\lambda^{b} \succeq_{a} \mu^{b}$. Again by [Br1, Lemma 2.5] there exists a sequence of elements $\mu^{b}=f_{(1)}, \ldots, f_{(k)}=\lambda^{b}$ in $\mathbb{Z}^{p \mid q}$ such that $f_{(i+1)} \downarrow f_{(i)}$ for $1 \leq i \leq k-1$. Since $\lambda^{b}, \mu^{b} \in \mathbb{Z}_{\geqslant}^{p \mid q}$, we can find a sequence of elements $\mu^{b}=g_{(1)}, \ldots, g_{(l)}=\lambda^{b}$ in $\mathbb{Z}_{\geqslant}^{p \mid q}$ such that $g_{(i+1)}=g_{(i)}-d_{-u_{i}}-d_{v_{i}}$ for some $1 \leq u_{i} \leq p$ and $1 \leq v_{i} \leq q$. Moreover by Lemma 3.5, $g_{(i)}=\nu_{(i)}^{b}$ for some $\nu_{(i)} \in \Lambda_{\mathbb{Z}^{\times}}^{\geqslant}$. This implies that $\nu_{(i)}+\beta_{i}=\nu_{(i+1)}$ with $\left(\nu_{(i)}, \beta_{i}\right)=0$ for some $\beta_{i} \in \Phi^{+}$. Therefore, $\lambda \succeq \mu$.

Corollary 3.7. For $\lambda, \mu \in \Lambda_{\frac{1}{2}+\mathbb{Z}}^{+}$, we have $\lambda \succcurlyeq \mu$ if and only if $\lambda^{\natural} \succeq_{a} \mu^{\natural}$, where $\lambda^{\natural}=\left(\lambda^{\sharp}\right)^{b}$.

\section{Dual Zuckerman functors}

Let $\mathfrak{b} \subseteq \mathfrak{p} \subseteq \mathfrak{g}$ be parabolic subalgebras with Levi subalgebra $\mathfrak{h} \subseteq \mathfrak{l} \subseteq \mathfrak{g}$, respectively. Let $\mathcal{H} C\left(\mathfrak{g}, \mathfrak{l}_{\overline{0}}\right)$ be the category of $\mathfrak{g}$-modules that are direct sums of finite-dimensional simple $\mathfrak{l}_{\overline{0}}$-modules, and let $\mathcal{H} \mathfrak{C}\left(\mathfrak{g}, \mathfrak{g}_{\overline{0}}\right)$ be defined similarly. Let $\mathcal{L}^{\mathfrak{g}, \mathfrak{l}}$ be the dual Zuckerman functor from $\mathcal{H} \mathcal{C}\left(\mathfrak{g}, \mathfrak{l}_{\overline{0}}\right)$ to $\mathcal{H} \mathcal{H}\left(\mathfrak{g}, \mathfrak{g}_{\overline{0}}\right)$ as in [San, Section 4], which is a right exact 
functor. For $i \geq 0$, we denote by $\mathcal{L}_{i}^{\mathfrak{g}, \mathfrak{l}}$ its $i$ th derived functor. For $M \in \mathcal{H} \mathfrak{C}\left(\mathfrak{g}, \mathfrak{l}_{\overline{0}}\right)$, we let

$$
\mathcal{E}^{\mathfrak{g}, \mathfrak{l}}(M):=\sum_{i \geq 0}(-1)^{i} \mathcal{L}_{i}^{\mathfrak{g}, \mathfrak{l}}(M)
$$

be the Euler characteristic of $M$, which is a virtual $\mathfrak{g}$-module.

By the same arguments as in [San, Sections 4 and 5], we can check the following.

Proposition 4.1. Let $\lambda \in \mathfrak{h}_{\overline{0}}^{*}$ such that the irreducible $\mathfrak{l}$-module $L(\mathfrak{l}, \lambda)$ with highest weight $\lambda$ is finite dimensional so that $M:=\operatorname{Ind}_{\mathfrak{p}}^{\mathfrak{g}} L(\mathfrak{l}, \lambda) \in \mathcal{H} \mathfrak{C}\left(\mathfrak{g}, \mathfrak{l}_{\overline{0}}\right)$.

(1) The $\mathfrak{g}$-module $\mathcal{L}_{0}^{\mathfrak{g}, \mathfrak{l}}(M)$ is the maximal finite-dimensional quotient of $M$.

(2) The $\mathfrak{g}$-module $\mathcal{L}_{i}^{\mathfrak{g}, \mathfrak{l}}(M)$ is finite dimensional for all $i \geq 0$, and $\mathcal{L}_{i}^{\mathfrak{g}, \mathfrak{l}}(M)=0$ for $i \gg 0$.

(3) Let I be the annihilator of $M$ in $U(\mathfrak{g})$. Then I annihilates every $\mathcal{L}_{i}^{\mathfrak{g}, \mathfrak{l}}(M)$ for all $i \geq 0$. In particular, all the $\mathfrak{g}$-modules $\mathcal{L}_{i}^{\mathfrak{g}, \mathfrak{l}}(M)$ have the same central character.

(4) The character of the Euler characteristic of $M$ is given by

$$
\operatorname{ch} \mathcal{E}^{\mathfrak{g}, \mathfrak{l}}(M)=D^{-1} \sum_{w \in W}(-1)^{\ell(w)} w\left(\frac{\operatorname{ch} L(\mathfrak{l}, \lambda)}{\prod_{\alpha \in \Phi^{+}\left(\mathfrak{l}_{\overline{1}}\right)}\left(1+e^{-\alpha}\right)}\right),
$$

where $\Phi^{+}\left(\mathfrak{l}_{\overline{1}}\right)$ denotes the set of positive roots of $\mathfrak{l}_{\overline{1}}$ and

$$
D_{\overline{0}}:=\prod_{\alpha \in \Phi_{\overline{0}}^{+}}\left(e^{\alpha / 2}-e^{-\alpha / 2}\right), \quad D_{\overline{1}}:=\prod_{\alpha \in \Phi_{\overline{1}}^{+}}\left(e^{\alpha / 2}+e^{-\alpha / 2}\right), \quad D:=\frac{D_{\overline{0}}}{D_{\overline{1}}} .
$$

For an exact sequence in $\mathcal{H} C\left(\mathfrak{g}, \mathfrak{l}_{\overline{0}}\right)$

$$
0 \longrightarrow M \longrightarrow E \longrightarrow N \longrightarrow 0,
$$

we have the following identity of virtual modules:

$$
\mathcal{E}^{\mathfrak{g}, \mathfrak{l}}(E)=\mathcal{E}^{\mathfrak{g}, \mathfrak{l}}(M)+\mathcal{E}^{\mathfrak{g}, \mathfrak{l}}(N) .
$$

Thus, Proposition 4.1(4) remains valid with $L(\mathfrak{l}, \lambda)$ replaced by a finite-dimensional l-module.

Remark 4.2. Let $\mathfrak{b} \subseteq \mathfrak{p} \subseteq \mathfrak{q} \subseteq \mathfrak{g}$ be parabolic subalgebras with Levi subalgebras $\mathfrak{h} \subseteq \mathfrak{l} \subseteq \mathfrak{k} \subseteq \mathfrak{g}$, respectively. Let $\mathfrak{u}^{\mathfrak{g}, \mathfrak{l}}$ and $\mathfrak{u}^{\mathfrak{g}, \mathfrak{k}}$ be the nilradicals of $\mathfrak{p}$ and $\mathfrak{q}$ such that $\mathfrak{p}=\mathfrak{l}+\mathfrak{u}^{\mathfrak{g}, \mathfrak{l}}$ and $\mathfrak{q}=\mathfrak{k}+\mathfrak{u}^{\mathfrak{g}, \mathfrak{k}}$, respectively. Let $\mathfrak{u}_{-}^{\mathfrak{g}, \mathfrak{l}}$ and $\mathfrak{u}_{-}^{\mathfrak{g}, \mathfrak{k}}$ denote the respective opposite nilradicals. Suppose that $M \in \mathcal{H} \mathfrak{C}\left(\mathfrak{l}, \mathfrak{l}_{\overline{0}}\right)$ which we extend to a p-module by $\mathfrak{u}^{\mathfrak{g}, \mathfrak{l}} M=0$. Consider the $\mathfrak{q}$-module $\operatorname{Ind}_{\mathfrak{p}}^{\mathfrak{q}} M=U\left(\mathfrak{k} \cap \mathfrak{u}_{-}^{\mathfrak{g}, \mathfrak{l}}\right) \otimes M$. Since $\left[\mathfrak{k}, \mathfrak{u}^{\mathfrak{g}, \mathfrak{k}}\right] \subseteq \mathfrak{u}^{\mathfrak{g}, \mathfrak{k}} \subseteq \mathfrak{u}^{\mathfrak{g}, \mathfrak{l}}$, we have $\mathfrak{u}^{\mathfrak{g}, \mathfrak{k}} \operatorname{Ind}_{\mathfrak{p}}^{\mathfrak{q}} M=0$. We conclude that $\operatorname{Ind}_{\mathfrak{p}}^{\mathfrak{q}} M$ is a $\mathfrak{k}$-module that is trivially extended to a $\mathfrak{q}$-module.

The following is the analogue of [PS2, Theorem 1] and [GS, Theorem 1] using the language of dual Zuckerman functor. 
Lemma 4.3. Let $\mathfrak{b} \subseteq \mathfrak{p} \subseteq \mathfrak{q} \subseteq \mathfrak{g}$ be parabolic subalgebras with Levi subalgebras $\mathfrak{h} \subseteq \mathfrak{l} \subseteq$ $\mathfrak{k} \subseteq \mathfrak{g}$, respectively. For $M \in \mathcal{H} C\left(\mathfrak{l}, \mathfrak{l}_{\overline{0}}\right)$, we have

$$
\mathcal{E}^{\mathfrak{g}, \mathfrak{l}}\left(\operatorname{Ind}_{\mathfrak{p}}^{\mathfrak{g}} M\right)=\mathcal{E}^{\mathfrak{g}, \mathfrak{k}}\left(\operatorname{Ind}_{\mathfrak{q}}^{\mathfrak{g}} \mathcal{E}^{\mathfrak{k}, \mathfrak{l}}\left(\operatorname{Ind}_{\mathfrak{p}}^{\mathfrak{q}} M\right)\right),
$$

where $M$ is regarded as a $\mathfrak{p}$-module by letting the nilradical act trivially.

Proof. First we have from the transitivity of dual Zuckerman functors (cf. [KV], Proposition 2.19]) that for $M \in \mathcal{H C}\left(\mathfrak{l}, \mathfrak{l}_{\overline{0}}\right)$

$$
\mathcal{E}^{\mathfrak{g}_{\overline{0}}, \mathfrak{l}_{\overline{0}}}\left(\operatorname{Ind}_{\mathfrak{p}_{\overline{0}}}^{\mathfrak{g}_{\overline{0}}} M\right)=\mathcal{E}^{\mathfrak{g}_{\overline{0}}, \mathfrak{k}_{\overline{0}}}\left(\operatorname{Ind}_{\mathfrak{q}_{\overline{0}}}^{\mathfrak{g}_{\overline{0}}} \mathcal{E}^{\mathfrak{k}_{\overline{0}}, \mathfrak{l}_{\overline{0}}}\left(\operatorname{Ind}_{\mathfrak{p}_{\overline{0}}}^{\mathfrak{q}_{\overline{0}}} M\right)\right),
$$

where we regard $M$ as an $\mathfrak{l}_{\overline{0}}$-module. Also from Mackey isomorphism (cf. [KV, Chapter II.5], we can deduce that for a finite-dimensional $\mathfrak{g}_{0}-$-module $N$

$$
N \otimes \mathcal{E}^{\mathfrak{g}_{\overline{0}}, \mathfrak{l}_{\overline{0}}}(M) \cong \mathcal{E}^{\mathfrak{g}_{\overline{0}}, l_{\overline{0}}}(N \otimes M),
$$

Let $\mathfrak{u}_{-}^{\mathfrak{g}, \mathfrak{k}}, \mathfrak{u}_{-}^{\mathfrak{k}, \mathfrak{l}}$, and $\mathfrak{u}_{-}^{\mathfrak{g}, \mathfrak{l}}$ be the opposite nilradicals such that $\mathfrak{g}=\mathfrak{q} \oplus \mathfrak{u}_{-}^{\mathfrak{g}, \mathfrak{k}}, \mathfrak{q}=\mathfrak{p} \oplus \mathfrak{u}_{-}^{\mathfrak{k}, \mathfrak{l}}$, and $\mathfrak{g}=\mathfrak{p} \oplus \mathfrak{u}_{-}^{\mathfrak{g}, \mathfrak{l}}$, respectively. Note that

$$
\begin{aligned}
& \operatorname{Res}_{\mathfrak{g}_{\overline{0}}}^{\mathfrak{g}} \mathcal{E}^{\mathfrak{g}, \mathfrak{l}}(U)=\mathcal{E}^{\mathfrak{g}_{\overline{0}}, \mathfrak{l}_{\overline{0}}}\left(\operatorname{Res}_{\mathfrak{g}_{\overline{0}}}^{\mathfrak{g}} U\right), \\
& \operatorname{Res}_{\mathfrak{g}_{\overline{0}}}^{\mathfrak{g}} \operatorname{Ind}_{\mathfrak{p}}^{\mathfrak{g}} V \cong \operatorname{Ind}_{\mathfrak{p}_{\overline{0}}}^{\mathfrak{g}_{\overline{0}}}\left(\Lambda\left(\left(\mathfrak{u}_{-}^{\mathfrak{g} l \mathfrak{l}}\right)_{\overline{1}}\right) \otimes \operatorname{Res}_{\mathfrak{l}_{\overline{0}}^{\mathfrak{l}}}^{\mathfrak{l}} V\right),
\end{aligned}
$$

for $U \in \mathcal{H} \mathcal{H}\left(\mathfrak{g}, \mathfrak{l}_{\overline{0}}\right)$ and $V \in \mathcal{H} \mathcal{C}\left(\mathfrak{l}, \mathfrak{l}_{\overline{0}}\right)$. For simplicity, let us omit the notation of Res if there is no confusion.

Now for $M \in \mathcal{H} \mathfrak{H}\left(\mathfrak{l}, \mathfrak{l}_{\overline{0}}\right)$, we have as $\mathfrak{g}_{\overline{0}}$-modules

$$
\begin{aligned}
& \mathcal{E}^{\mathfrak{g}, \mathfrak{k}}\left(\operatorname{Ind}_{\mathfrak{q}}^{\mathfrak{g}} \mathcal{E}^{\mathfrak{k}, \mathfrak{l}}\left(\operatorname{Ind}_{\mathfrak{p}}^{\mathfrak{q}} M\right)\right) \\
& \cong \mathcal{E}^{\mathfrak{g}_{\overline{0}}, \mathfrak{k}_{\overline{0}}}\left(\operatorname{Ind}_{\mathfrak{q}_{\overline{0}}}^{\mathfrak{g}_{\overline{0}}}\left(\Lambda\left(\left(\mathfrak{u}_{-}^{\mathfrak{g}, \mathfrak{k}}\right)_{\overline{1}}\right) \otimes \mathcal{E}^{\mathfrak{k}_{\overline{0}}, \mathfrak{l}_{\overline{0}}}\left(\operatorname{Ind}_{\mathfrak{p}_{\overline{0}}}^{\mathfrak{q}_{\overline{0}}}\left(\Lambda\left(\left(\mathfrak{u}_{-}^{\mathfrak{k}, \mathfrak{l}}\right)_{\overline{1}}\right) \otimes M\right)\right)\right)\right) \quad \text { by (4.4) } \\
& \cong \mathcal{E}^{\mathfrak{g}_{\overline{0}}, \mathfrak{k}_{\overline{0}}}\left(\operatorname{Ind}_{\mathfrak{q}_{\overline{0}}}^{\mathfrak{g}_{\overline{0}}}\left(\mathcal{E}^{\mathfrak{k}_{\overline{0}}, \mathfrak{l}_{\overline{0}}}\left(\Lambda\left(\left(\mathfrak{u}_{-}^{\mathfrak{g}, \mathfrak{k}}\right)_{\overline{1}}\right) \otimes \operatorname{Ind}_{\mathfrak{p}_{\overline{0}}}^{\mathfrak{q}_{\overline{0}}}\left(\Lambda\left(\left(\mathfrak{u}_{-}^{\mathfrak{k}, \mathfrak{l}}\right)_{\overline{1}}\right) \otimes M\right)\right)\right)\right) \quad \text { by (4.3) } \\
& \cong \mathcal{E}^{\mathfrak{g}_{\overline{0}}, \mathfrak{k}_{\overline{0}}}\left(\operatorname{Ind}_{\mathfrak{q}_{\overline{0}}}^{\mathfrak{g}_{\overline{0}}}\left(\mathcal{E}^{\mathfrak{k}_{\overline{0}}, \mathfrak{l}_{\overline{0}}}\left(\operatorname{Ind}_{\mathfrak{p}_{\overline{0}}}^{\mathfrak{q}_{\overline{0}}}\left(\Lambda\left(\left(\mathfrak{u}_{-}^{\mathfrak{g}, \mathfrak{k}}\right)_{\overline{1}}\right) \otimes \Lambda\left(\left(\mathfrak{u}_{-}^{\mathfrak{k}, \mathfrak{l}}\right)_{\overline{1}}\right) \otimes M\right)\right)\right)\right) \\
& \cong \mathcal{E}^{\mathfrak{g}_{\overline{0}}, \mathfrak{k}_{\overline{0}}}\left(\operatorname{Ind}_{\mathfrak{q}_{\overline{0}}}^{\mathfrak{g}_{\overline{0}}}\left(\mathcal{E}^{\mathfrak{k}_{\overline{0}}, \mathfrak{l}_{\overline{0}}}\left(\operatorname{Ind}_{\mathfrak{p}_{\overline{0}}}^{\mathfrak{q}_{\overline{0}}}\left(\Lambda\left(\left(\mathfrak{u}_{-}^{\mathfrak{g}, \mathfrak{l}}\right)_{\overline{1}}\right) \otimes M\right)\right)\right)\right) \\
& \cong \mathcal{E}^{\mathfrak{g}_{\overline{0}}, \mathfrak{l}_{\overline{0}}}\left(\operatorname{Ind}_{\mathfrak{p}_{\overline{0}}}^{\mathfrak{g}_{\overline{0}}}\left(\Lambda\left(\left(\mathfrak{u}_{-}^{\mathfrak{g}, \mathfrak{l}}\right)_{\overline{1}}\right) \otimes M\right)\right) \quad \text { by (4.2) } \\
& \cong \mathcal{E}^{\mathfrak{g}, \mathfrak{l}}\left(\operatorname{Ind}_{\mathfrak{q}}^{\mathfrak{g}} M\right) \text {. }
\end{aligned}
$$

The proof is complete.

For $\lambda \in \Lambda^{+}$, we may understand $\lambda$ as a weight for $\mathfrak{l}$ or $\mathfrak{k}$. We regard the irreducible $\mathfrak{l}$ module $L(\mathfrak{l}, \lambda)$ with highest weight $\lambda$ as an irreducible $\mathfrak{p}$-module by letting the nilradical act trivially. Similarly, we have the irreducible $\mathfrak{k}$-module $L(\mathfrak{k}, \lambda)$ which can be viewed as an irreducible $\mathfrak{q}$-module. For $\lambda, \mu \in \Lambda^{+}$, we let

$$
m_{i}^{\mathfrak{q}, \mathfrak{p}}(\lambda, \mu):=\left[\mathcal{L}_{i}^{\mathfrak{k}, \mathfrak{l}}\left(\operatorname{Ind}_{\mathfrak{p}}^{\mathfrak{q}} L(\mathfrak{l}, \lambda)\right): L(\mathfrak{k}, \mu)\right]
$$


the multiplicity of the irreducible $\mathfrak{k}$-module $L(\mathfrak{k}, \mu)$ in $\mathcal{L}_{i}^{\mathfrak{k}, \mathfrak{l}}\left(\operatorname{Ind}_{\mathfrak{p}}^{\mathfrak{q}} L(\mathfrak{l}, \lambda)\right)$.

Let $\lambda \in \Lambda^{+}$be given. Put

$$
\mathfrak{l}(\lambda)=\mathfrak{h} \oplus \bigoplus_{\alpha \in \Phi^{+}(\lambda)}\left(\mathfrak{g}_{\alpha} \oplus \mathfrak{g}_{-\alpha}\right), \quad \mathfrak{p}(\lambda)=\mathfrak{l}(\lambda)+\mathfrak{b},
$$

where $\Phi^{+}(\lambda)=\left\{\varepsilon_{i}-\varepsilon_{j} \mid \lambda_{i}=\lambda_{j}(i<j)\right\}$ and $\mathfrak{g}_{\alpha}$ is the root space of $\mathfrak{g}$ corresponding to $\alpha$. Note that $\mathfrak{l}(\lambda)=\mathfrak{h}$ for $\lambda \in \Lambda_{\frac{1}{2}+\mathbb{Z}}^{+}$.

Let $E(\lambda):=\mathcal{E}^{\mathfrak{g}, \mathfrak{l}(\lambda)}\left(\operatorname{Ind}_{\mathfrak{p}(\lambda)}^{\mathfrak{g}} W_{\lambda}\right)^{2}$ and here $W_{\lambda}$ is regarded as an irreducible $\mathfrak{r}(\lambda)$ module. We have by Proposition 4.1(4):

$$
\operatorname{ch} E(\lambda)=2^{\lceil\ell(\lambda) / 2\rceil} D^{-1} \sum_{w \in W}(-1)^{\ell(w)} w\left(\frac{e^{\lambda}}{\prod_{\beta \in \Phi^{+}(\lambda)}\left(1+e^{-\beta}\right)}\right) .
$$

It is well-known that we have the following two bases in $K\left(\mathcal{O}_{\epsilon+\mathbb{Z}}^{\text {fin }}\right)$ :

$$
\left\{[E(\lambda)] \mid \lambda \in \Lambda_{\epsilon+\mathbb{Z}}^{+}\right\}, \quad\left\{[L(\lambda)] \mid \lambda \in \Lambda_{\epsilon+\mathbb{Z}}^{+}\right\} .
$$

We thus have

$$
[E(\lambda)]=\sum_{\mu \in \Lambda_{\epsilon+\mathbb{Z}}^{+}} a_{\lambda \mu}[L(\mu)]
$$

where $a_{\lambda \mu}=\sum_{i \geq 0}(-1)^{i} m_{i}^{\mathfrak{g}, \mathfrak{p}(\lambda)}(\lambda, \mu)$.

Let

$$
\mathfrak{p}(\lambda)=\mathfrak{p}_{0} \subseteq \mathfrak{p}_{1} \subseteq \cdots \subseteq \mathfrak{p}_{k-1} \subseteq \mathfrak{p}_{k}=\mathfrak{g}
$$

be a sequence of parabolic subalgebras with respective sequence of Levi subalgebras

$$
\mathfrak{l}(\lambda)=\mathfrak{l}_{0} \subseteq \mathfrak{l}_{1} \subseteq \cdots \subseteq \mathfrak{l}_{k-1} \subseteq \mathfrak{l}_{k}=\mathfrak{g} .
$$

We have

$$
\operatorname{Ind}_{\mathfrak{p}(\lambda)}^{\mathfrak{g}} W_{\lambda}=\operatorname{Ind}_{\mathfrak{p}_{k-1}}^{\mathfrak{p}_{k}} \cdots \operatorname{Ind}_{\mathfrak{p}_{1}}^{\mathfrak{p}_{2}} \operatorname{Ind}_{\mathfrak{p}_{0}}^{\mathfrak{p}_{1}} W_{\lambda}
$$

If we put $\mathcal{E}^{s, s-1}=\mathcal{E}^{\mathfrak{l}_{s}, \mathfrak{l}_{s-1}}, \operatorname{Ind}_{s-1}^{s}=\operatorname{Ind}_{\mathfrak{p}_{s-1}}^{\mathfrak{p}_{s}}$, and $m_{i}^{s, s-1}(\mu, \nu)=m_{i}^{\mathfrak{p}_{s}, \mathfrak{p}_{s-1}}(\mu, \nu)$ for $1 \leq s \leq k, i \geq 0$, and $\mu, \nu \in \Lambda^{+}$, then we have by Lemma 4.3

$$
\begin{aligned}
& E(\lambda) \\
& =\mathcal{E}^{k, 0}\left(\operatorname{Ind}_{k-1}^{k} \cdots \operatorname{Ind}_{1}^{2} \operatorname{Ind}_{0}^{1} W_{\lambda}\right) \\
& =\mathcal{E}^{k, k-1}\left(\operatorname{Ind}_{k-1}^{k} \mathcal{E}^{k-1, k-2}\left(\cdots \mathcal{E}^{2,1}\left(\operatorname{Ind}_{1}^{2} \mathcal{E}^{1,0}\left(\operatorname{Ind}_{0}^{1} W_{\lambda}\right)\right) \cdots\right)\right) \\
& =\mathcal{E}^{k, k-1}\left(\cdots \mathcal{E}^{2,1}\left(\operatorname{Ind}_{1}^{2} \sum_{i_{1} \geq 0, \lambda^{(1)}}(-1)^{i_{1}} m_{i_{1}}^{1,0}\left(\lambda, \lambda^{(1)}\right) L\left(\mathfrak{l}_{1}, \lambda^{(1)}\right)\right) \cdots\right)
\end{aligned}
$$




$$
\begin{aligned}
& =\varepsilon^{k, k-1}\left(\cdots \varepsilon^{3,2}\left(\operatorname{Ind}_{2}^{3} \sum_{\substack{i_{1}, i_{2} \geq 0 \\
\lambda^{(1)}, \lambda^{(2)}}}(-1)^{i_{1}+i_{2}} m_{i_{1}}^{1,0}\left(\lambda, \lambda^{(1)}\right) m_{i_{2}}^{2,1}\left(\lambda^{(1)}, \lambda^{(2)}\right) L\left(\mathfrak{l}_{2}, \lambda^{(2)}\right)\right) \cdots\right) \\
& =\cdots \\
& =\sum_{\substack{i_{1}, \ldots, i_{k} \geq 0 \\
\lambda^{(1)}, \ldots, \lambda^{(k)}}}(-1)^{i_{1}+\cdots+i_{k}} m_{i_{1}}^{1,0}\left(\lambda, \lambda^{(1)}\right) m_{i_{2}}^{2,1}\left(\lambda^{(1)}, \lambda^{(2)}\right) \cdots m_{i_{k}}^{k, k-1}\left(\lambda^{(k-1)}, \lambda^{(k)}\right) L\left(\lambda^{(k)}\right) .
\end{aligned}
$$

Comparing with (4.7) we get the following formula for $a_{\lambda \mu}$ :

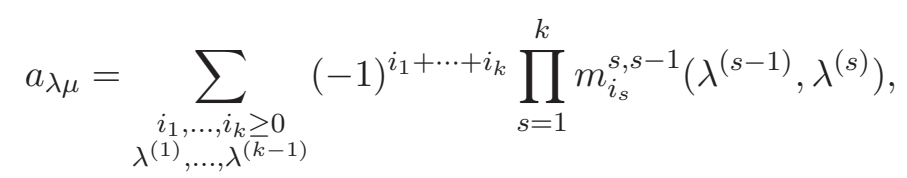

where $\lambda^{(0)}=\lambda$ and $\lambda^{(k)}=\mu$. Note that for different $\mu$ 's, we can choose in principle different sequences of parabolic subalgebras. This way we obtain a method to compute the coefficients $a_{\lambda \mu}$, if we can find a sequence of parabolic subalgebras as in (4.8) for which we can compute the multiplicity $m_{i}^{s, s-1}(\nu, \eta)$, for all relevant $\nu, \eta$. This is the essence of the algorithm in [PS2, which we shall use in the next section.

\section{Penkov-Serganova Algorithm}

Below is an analogue of the "typical lemma" ([GS, Lemma 5]) for the queer Lie superalgebra. Although our proof below is algebraic in nature, it is inspired by loc. cit. We mention that this lemma already appeared in [PS2, Theorem 2].

Proposition 5.1. Let $\lambda=\sum_{i=1}^{n} \lambda_{i} \varepsilon_{i} \in \Lambda^{+}$be given. Suppose that $\mathfrak{l}$ is a Levi subalgebra that contains every positive root atypical to $\lambda$. Then we have

$$
\mathcal{L}_{i}^{\mathfrak{g}, \mathfrak{l}}\left(\operatorname{Ind}_{\mathfrak{p}}^{\mathfrak{g}} L(\mathfrak{l}, \lambda)\right)= \begin{cases}L(\lambda), & \text { if } i=0 \\ 0, & \text { if } i>0\end{cases}
$$

In particular, we have $\operatorname{ch}^{\mathfrak{g}, \mathfrak{l}}\left(\operatorname{Ind}_{\mathfrak{p}}^{\mathfrak{g}} L(\mathfrak{l}, \lambda)\right)=\operatorname{ch} L(\lambda)$.

Proof. First, we take a coweight $h_{\mathfrak{l}}=\sum_{i=1}^{n} c_{i} h_{i} \in \mathfrak{h}_{\overline{0}}$ satisfying

$$
\begin{cases}c_{i}-c_{i+1}=0, & \text { if } \varepsilon_{i}-\varepsilon_{i+1} \in \Phi^{+}\left(\mathfrak{l}_{\overline{0}}\right), \\ c_{i}-c_{i+1}=1, & \text { if } \varepsilon_{i}-\varepsilon_{i+1} \notin \Phi^{+}\left(\mathfrak{l}_{\overline{0}}\right),\end{cases}
$$

for $1 \leq i \leq n-1$. Then $\Phi(\mathfrak{l})=\left\{\alpha \in \Phi \mid\left\langle\alpha, h_{\mathfrak{l}}\right\rangle=0\right\}$ and $\Phi(\mathfrak{p})=\left\{\alpha \in \Phi \mid\left\langle\alpha, h_{\mathfrak{l}}\right\rangle \geq 0\right\}$, where $\Phi, \Phi(\mathfrak{l})$, and $\Phi(\mathfrak{p})$ denote the set of roots of $\mathfrak{g}, \mathfrak{l}$, and $\mathfrak{p}$, respectively. Denote by $W_{\mathfrak{l}}$ the Weyl group of $\mathfrak{l}$.

Let $\mu \in \Lambda^{+}$and suppose that $L(\mu)$ occurs in $\mathcal{L}_{i}^{\mathfrak{g}, \mathfrak{l}}\left(\operatorname{Ind}_{\mathfrak{p}}^{\mathfrak{g}} L(\mathfrak{l}, \lambda)\right)$ for some $i \geq 0$. Now, observe that we have $L(\mathfrak{l}, \lambda)=U\left(\mathfrak{l}_{\overline{1}} \cap \mathfrak{n}_{-}\right) U\left(\mathfrak{l}_{\overline{0}} \cap \mathfrak{n}_{-}\right) W_{\lambda}=U\left(\mathfrak{l}_{\overline{1}} \cap \mathfrak{n}_{-}\right) E$, where we recall 
that $\mathfrak{n}_{-}$is the opposite nilradical of $\mathfrak{b}$, and $E$, as an $\mathfrak{l}_{\overline{0}}$-module, is a direct sum of copies of the irreducible $\mathfrak{l}_{\hat{0}}$-module of highest weight $\lambda$. Thus, by [Ger, Lemma 1.3.3], we have

$$
\mu=w\left(\lambda-\sum_{\alpha \in J} \alpha+\rho_{\overline{0}}\right)-\rho_{\overline{0}}
$$

for some $w \in W$ with $\ell(w)=i$ and $J \subseteq \Phi_{\overline{1}}^{+}$. Since $\rho_{\overline{0}}=\rho_{\overline{1}}$ and in the expression $-\sum_{\alpha \in J} \alpha+\rho_{\overline{1}}$ every $\alpha \in \Phi_{\overline{1}}^{+}$appears exactly once with coefficient $\frac{1}{2}$ or $-\frac{1}{2}$, we have $w\left(-\sum_{\alpha \in J} \alpha+\rho_{\overline{1}}\right)-\rho_{\overline{1}}=-\sum_{\alpha \in K} \alpha$, for some $K \subseteq \Phi_{\overline{1}}^{+}$, and hence

$$
\mu=w(\lambda)-\sum_{\alpha \in K} \alpha
$$

Note that $\lambda-w(\lambda)=\sum_{\alpha \in \Phi_{\overline{0}}^{+}} k_{\alpha} \alpha$ for some $k_{\alpha} \in \mathbb{Z}_{+}$since $\lambda$ is a $\mathfrak{g}_{\overline{0}}$-dominant weight. So we have

$$
\left\langle\mu, h_{\mathfrak{l}}\right\rangle \leq\left\langle w(\lambda), h_{\mathfrak{l}}\right\rangle \leq\left\langle\lambda, h_{\mathfrak{l}}\right\rangle
$$

Suppose that the degree of atypicality of $\lambda$ is $r$, and let $S_{\lambda}$ be a set of mutually orthogonal $r$ even positive roots atypical to $\lambda$. Since $\chi_{\mu}=\chi_{\lambda}$ by Proposition 4.1(3), we have by the linkage principle for $\mathfrak{g}$ ([Sv2], see also [CW, Theorem 2.48])

$$
v(\mu)=\lambda+\sum_{\alpha \in S_{\lambda}} c_{\alpha} \alpha,
$$

for some $c_{\alpha}$ and $v \in W$. Since $\left\langle v(\mu), h_{\mathfrak{l}}\right\rangle \leq\left\langle\mu, h_{\mathfrak{l}}\right\rangle$ as in (5.3), we have

$$
\left\langle\mu, h_{\mathfrak{l}}\right\rangle \geq\left\langle v(\mu), h_{\mathfrak{l}}\right\rangle=\left\langle\lambda, h_{\mathfrak{l}}\right\rangle+\sum_{\alpha \in S_{\lambda}} c_{\alpha}\left\langle\alpha, h_{\mathfrak{l}}\right\rangle=\left\langle\lambda, h_{\mathfrak{l}}\right\rangle
$$

So we have $\left\langle\mu, h_{\mathfrak{l}}\right\rangle=\left\langle w(\lambda), h_{\mathfrak{l}}\right\rangle=\left\langle\lambda, h_{\mathfrak{l}}\right\rangle$ by (5.3) and (5.4), and $K \subset \Phi^{+}(\mathfrak{l})$.

Since $\left\langle w(\lambda), h_{\mathfrak{l}}\right\rangle=\left\langle\lambda, h_{\mathfrak{l}}\right\rangle$ and $\langle\lambda, \beta\rangle>0$ for $\beta \notin \Phi^{+}\left(\mathfrak{l}_{\overline{0}}\right)$, we conclude that $w \in W_{\mathfrak{l}}$, and then $J \subseteq \Phi_{\overline{1}}^{+}(\mathfrak{l})$. By construction of $\mu$ (cf. [Ger, Lemma 1.3.3]), we see that $\lambda-\sum_{\alpha \in J} \alpha$ is a weight of $L(\mathfrak{l}, \lambda)$ and $\mathfrak{l}_{\overline{0}}$-dominant. Hence we have $w=i d$, and in particular, $\mathcal{L}_{i}^{\mathfrak{g}, \mathfrak{l}}\left(\operatorname{Ind}_{\mathfrak{p}}^{\mathfrak{g}} L(\mathfrak{l}, \lambda)\right)=0$ for $i>0$. Finally, since $\mu=\lambda-\sum_{\alpha \in J} \alpha$ is a weight of $L(\mathfrak{l}, \lambda)$ and $L(\mu)$ is a composition factor of $\operatorname{Ind}_{\mathfrak{p}}^{\mathfrak{g}} L(\mathfrak{l}, \lambda)$ by Proposition $4.1(1)$, we have $\mu=\lambda$. The proof completes.

Let $\lambda \in \Lambda^{+}$and suppose that $\mathfrak{p}$ is a parabolic subalgebra with Levi subalgebra $\mathfrak{l} \cong \mathfrak{q}(k)$ that contains every positive root atypical to $\lambda$. Recalling $\mathfrak{l}(\lambda)$ and $\mathfrak{p}(\lambda)$ from (4.5), we conclude that $\mathfrak{l}(\lambda) \subseteq \mathfrak{l}$ and $\mathfrak{p}(\lambda) \subseteq \mathfrak{p}$.

Suppose that

$$
\mathcal{E}^{\mathfrak{g}, \mathfrak{l}(\lambda)}\left(\operatorname{Ind}_{\mathfrak{p}(\lambda)}^{\mathfrak{g}} W_{\lambda}\right)=\sum_{\mu} a_{\lambda \mu} L(\mu), \quad \mathcal{E}^{\mathfrak{l}, \mathfrak{l}(\lambda)}\left(\operatorname{Ind}_{\mathfrak{p}(\lambda)}^{\mathfrak{l}} W_{\lambda}\right)=\sum_{\mu} a_{\lambda \mu}^{\prime} L(\mathfrak{l}, \mu) .
$$


Since $\lambda \succcurlyeq \mu$ above, we observe that $\mathfrak{l}$ also contains all positive root atypical to $\lambda$. We compute, using Lemma 4.3 and Proposition 5.1

$$
\begin{aligned}
\sum_{\mu} a_{\lambda \mu} L(\mu) & =\mathcal{E}^{\mathfrak{g}, \mathfrak{l}(\lambda)}\left(\operatorname{Ind}_{\mathfrak{p}(\lambda)}^{\mathfrak{g}} W_{\lambda}\right)=\mathcal{E}^{\mathfrak{g}, \mathfrak{l}}\left(\operatorname{Ind}_{\mathfrak{p}}^{\mathfrak{g}} \mathcal{E}^{\mathfrak{l}, \mathfrak{l}(\lambda)}\left(\operatorname{Ind}_{\mathfrak{p}(\lambda)}^{\mathfrak{p}} W_{\lambda}\right)\right) \\
& =\mathcal{E}^{\mathfrak{g}, \mathfrak{l}}\left(\operatorname{Ind}_{\mathfrak{p}}^{\mathfrak{g}} \sum_{\mu} a_{\lambda \mu}^{\prime} L(\mathfrak{l}, \mu)\right)=\sum_{\mu} a_{\lambda \mu}^{\prime} L(\mu) .
\end{aligned}
$$

We conclude that

$$
a_{\lambda \mu}=a_{\lambda \mu}^{\prime}
$$

The following is a crucial observation in the rank reduction algorithm in PS2, Theorem 3].

Lemma 5.2. Suppose that $\mathfrak{b} \subseteq \mathfrak{p} \subseteq \mathfrak{q} \subseteq \mathfrak{g}$ are parabolic subalgebras with Levi subalgebras $\mathfrak{h} \subseteq \mathfrak{l} \subseteq \mathfrak{k} \subseteq \mathfrak{g}$ respectively such that $\mathfrak{p} \cap \mathfrak{k}$ is the maximal parabolic subalgebra of $\mathfrak{k}$ corresponding to removing the left-most node in the Dynkin diagram of $\mathfrak{k}_{\overline{0}}$. Then for $\lambda, \mu \in \Lambda_{\frac{1}{2}+\mathbb{Z}}^{+}$and $i \geq 0$, we have

$$
m_{i}^{\mathfrak{q}, \mathfrak{p}}(\lambda, \mu)=m_{i}^{\mathfrak{q}, \mathfrak{p}}\left(\lambda^{\sharp}, \mu^{\sharp}\right) .
$$

Proof. In light of (5.5) we only need to consider weights of the form that appear in PS2, Theorem 3].

We apply the reduction algorithm $\left[\overline{\mathrm{PS} 2}\right.$, Theorem 3] to both $m_{i}^{\mathfrak{q}, \mathfrak{p}}(\lambda, \mu)$ and $m_{i}^{\mathfrak{q}, \mathfrak{p}}\left(\lambda^{\sharp}, \mu^{\sharp}\right)$, and compare their values. Note that in either reduction algorithm the case [PS2, (1.11)] cannot occur. Applying [PS2, Theorem 3] once to $m_{i}^{\mathfrak{q}, \mathfrak{p}}(\lambda, \mu)$, we have

$$
m_{i}^{\mathfrak{q}, \mathfrak{p}}(\lambda, \mu)=m_{i^{\prime}}^{\mathfrak{q}^{\prime}, \mathfrak{p}^{\prime}}\left(\lambda^{\prime}, \mu^{\prime}\right),
$$

for some $\mathfrak{p}^{\prime}$ and $\mathfrak{q}^{\prime}$ with ranks smaller than those of $\mathfrak{p}$ and $\mathfrak{q}$, respectively, and some $i^{\prime} \geq 0$. Moreover, applying [PS2, Theorem 3] to $m_{i}^{\mathfrak{q}, \mathfrak{p}}\left(\lambda^{\sharp}, \mu^{\sharp}\right)$ gives

$$
m_{i}^{\mathfrak{q}, \mathfrak{p}}\left(\lambda^{\sharp}, \mu^{\sharp}\right)=m_{i^{\prime}}^{\mathfrak{q}^{\prime}, \mathfrak{p}^{\prime}}\left(\left(\lambda^{\prime}\right)^{\sharp},\left(\mu^{\prime}\right)^{\sharp}\right),
$$

with the same $\mathfrak{p}^{\prime}, \mathfrak{q}^{\prime}$, and $i^{\prime}$ as in (5.6). Now, we apply the reduction algorithm as far as possible so that $m_{i^{\prime}}^{\mathfrak{q}^{\prime}, \mathfrak{p}^{\prime}}\left(\lambda^{\prime}, \mu^{\prime}\right)$ corresponds to either [PS2, Theorem 3(a)(1.8)] or [PS2, Theorem 4(c)], which is equivalent to the case when $m_{i^{\prime}}^{\mathfrak{q}^{\prime} \mathfrak{p}^{\prime}}\left(\left(\lambda^{\prime}\right)^{\sharp},\left(\mu^{\prime}\right)^{\sharp}\right)$ corresponds to [PS2, Theorem 3(a)(1.8)] or [PS2, Theorem 4(b)(1.16)] with no zeroes in $\left(\lambda^{\prime}\right)^{\sharp}$ and $\left(\mu^{\prime}\right)^{\sharp}$, respectively. In this case, we can also see that

$$
m_{i^{\prime}}^{\mathfrak{q}^{\prime}, \mathfrak{p}^{\prime}}\left(\lambda^{\prime}, \mu^{\prime}\right)=m_{i^{\prime}}^{\mathfrak{q}^{\prime}, \mathfrak{p}^{\prime}}\left(\left(\lambda^{\prime}\right)^{\sharp},\left(\mu^{\prime}\right)^{\sharp}\right) \in\{0,1\} .
$$

Therefore, it follows from (5.6) $-(5.8)$ that $m_{i}^{\mathfrak{q}, \mathfrak{p}}(\lambda, \mu)=m_{i}^{\mathfrak{q}, \mathfrak{p}}\left(\lambda^{\sharp}, \mu^{\sharp}\right)$.

Theorem 5.3. For $\lambda, \mu \in \Lambda_{\frac{1}{2}+\mathbb{Z}}^{+}$, we have

$$
a_{\lambda \mu}=a_{\lambda^{\sharp} \mu^{\sharp}} .
$$


Proof. We fix the sequence of parabolic subalgebras of $\mathfrak{g}$ of the form (4.8) such that, for each $s, \mathfrak{p}_{s-1} \cap \mathfrak{l}_{s}$ is the maximal parabolic subalgebra of $\mathfrak{l}_{s}$ corresponding to the removal of the left-most node of the Dynkin diagram of $\left(\mathfrak{l}_{s}\right)_{\overline{0}}$. Now we apply Lemma 5.2 to (4.9).

\section{BRUNDAN-KAZHDAN-LUSZTig THEORY}

Let us first briefly recall the results in $[\mathrm{Br} 2$. Let $q$ be an indeterminate. Let $\mathscr{U}=$ $U_{q}\left(\mathfrak{b}_{\infty}\right)$ be the quantum group associated to the Lie algebra of type $\mathfrak{b}_{\infty}$, and let $\mathscr{V}$ be its natural representation with basis $\left\{v_{a} \mid a \in \mathbb{Z}\right\}$. Fix $n \geq 1$. The space $\mathscr{T}^{n}:=\mathscr{V}^{\otimes n}$ is naturally a $\mathscr{U}$-module with standard monomial basis $\left\{N_{\lambda}:=v_{\lambda_{1}} \otimes \cdots \otimes v_{\lambda_{n}} \mid \lambda=\right.$ $\left.\sum_{i=1}^{n} \lambda_{i} \varepsilon_{i} \in \Lambda_{\mathbb{Z}}\right\}$. For $\lambda \in \Lambda_{\mathbb{Z}}$, set $M_{\lambda}:=\left(q+q^{-1}\right)^{z(\lambda)} N_{\lambda}$, where $z(\lambda)=n-\ell(\lambda)$. We can define a topological completion $\widehat{\mathscr{T}}^{n}$, compatible with the Bruhat ordering, on which one has a bar involution -. There exist unique bar-invariant topological bases called the canonical and dual canonical basis, denoted by $\left\{T_{\lambda} \mid \lambda \in \Lambda_{\mathbb{Z}}\right\}$ and $\left\{L_{\lambda} \mid \lambda \in \Lambda_{\mathbb{Z}}\right\}$, respectively [Br2, Theorem 2.22]. We have

$$
T_{\lambda}=\sum_{\mu} t_{\mu \lambda}(q) N_{\mu}, \quad L_{\lambda}=\sum_{\mu} \ell_{\mu \lambda}(q) M_{\mu}
$$

for some polynomials $t_{\mu \lambda}(q) \in \mathbb{Z}[q]$ and $\ell_{\mu \lambda}(q) \in \mathbb{Z}\left[q^{-1}\right]$ such that $t_{\mu \lambda}(q)=\ell_{\mu \lambda}(q)=0$, unless $\lambda \succeq \mu$ and $t_{\lambda \lambda}(q)=\ell_{\lambda \lambda}(q)=1$.

Let

$$
\mathscr{F}^{n}:=\bigwedge^{n} \mathscr{V}
$$

be the $q$-deformed $n$th exterior power of $\mathscr{V}$ of type $\mathfrak{b}_{\infty}$ as in [Br2, Section 3], which is a $\mathscr{U}$-module. This exterior module was first constructed in [JMO. The space $\mathscr{F}^{n}$ has a basis $\left\{F_{\lambda}:=v_{\lambda_{n}} \wedge \cdots \wedge v_{\lambda_{1}} \mid \lambda \in \Lambda_{\mathbb{Z}}^{+}\right\}$, and it admits a topological completion $\widehat{\mathscr{F}}^{n}$. The bar involution on $\widehat{\mathscr{T}}^{n}$ induces a bar involution on $\widehat{\mathscr{F}}^{n}$, and there exists a unique bar-invariant topological basis $\left\{U_{\lambda} \mid \lambda \in \Lambda_{\mathbb{Z}}^{+}\right\}$called the canonical basis $[\mathrm{Br2}$, Theorem 3.5], where we have

$$
U_{\lambda}=\sum_{\mu \in \Lambda_{\mathbb{Z}}^{+}} u_{\mu \lambda}(q) F_{\mu},
$$

for some $u_{\mu \lambda} \in \mathbb{Z}[q]$ such that $u_{\mu \lambda}(q) \neq 0$ unless $\mu \succeq \lambda$ and $u_{\lambda \lambda}(q)=1$. If $\pi: \mathscr{T}^{n} \rightarrow \mathscr{F}^{n}$ is the canonical projection map, then we have $\pi\left(T_{w_{0} \lambda}\right)=U_{\lambda}$ if $\lambda \in \Lambda_{\mathbb{Z}}^{+}$, and 0 otherwise, where $w_{0}$ is the longest element in $W$.

For $\lambda \in \Lambda_{\mathbb{Z}}^{+}$we define

$$
E_{\lambda}:=\sum_{\mu \in \Lambda_{\mathbb{Z}}^{+}, \lambda \succeq \mu} u_{-w_{0} \lambda,-w_{0} \mu}\left(q^{-1}\right) L_{\mu},
$$


and let

$$
\mathscr{E}^{n}:=\sum_{\lambda \in \Lambda_{\mathbb{Z}}^{+}} \mathbb{Q}(q) E_{\lambda} \subset \widehat{\mathscr{T}}^{n} .
$$

One can show [Br2, Theorem 3.16] that $\left\{L_{\lambda} \mid \lambda \in \Lambda_{\mathbb{Z}}^{+}\right\}$is the unique bar-invariant basis of $\mathscr{E}^{n}$, and $L_{\lambda}=\sum_{\mu \in \Lambda_{\mathbb{Z}}^{+}} \ell_{\mu \lambda}(q) E_{\mu}$ for $\lambda \in \Lambda_{\mathbb{Z}}^{+}$. Put

$$
\begin{aligned}
& \mathscr{E}_{\mathbb{Z}\left[q, q^{-1}\right]}:=\sum_{\lambda \in \Lambda_{\mathbb{Z}}^{+}} \mathbb{Z}\left[q, q^{-1}\right] L_{\lambda}=\sum_{\lambda \in \Lambda_{\mathbb{Z}}^{+}} \mathbb{Z}\left[q, q^{-1}\right] E_{\lambda}, \\
& \mathscr{E}_{\mathbb{Z}}:=\mathbb{Z} \otimes_{\mathbb{Z}\left[q, q^{-1}\right]} \mathscr{E}_{\mathbb{Z}\left[q, q^{-1}\right]},
\end{aligned}
$$

where $\mathbb{Z}$ is the right $\mathbb{Z}\left[q, q^{-1}\right]$-module with $q$ acting on $\mathbb{Z}$ as 1 . Let $E_{\lambda}(1)=1 \otimes E_{\lambda}$ and $L_{\lambda}(1)=1 \otimes L_{\lambda} \in \mathscr{E}_{\mathbb{Z}}$ for $\lambda \in \Lambda_{\mathbb{Z}}^{+}$. The following is the main result in [Br2]:

Theorem 6.1 (Theorem 4.52 in $[\overline{\mathrm{Br} 2}])$. Let $\Psi: K\left(\mathcal{O}_{\mathbb{Z}}^{\text {fin }}\right) \rightarrow \mathscr{E}_{\mathbb{Z}}$ be the $\mathbb{Z}$-linear isomorphism defined by

$$
[E(\lambda)] \longmapsto E_{\lambda}(1) \quad\left(\lambda \in \Lambda_{\mathbb{Z}}^{+}\right)
$$

Then $\Psi([L(\lambda)])=L_{\lambda}(1)$, for all $\lambda \in \Lambda_{\mathbb{Z}}^{+}$.

Now we consider $K\left(\mathcal{O}_{\frac{1}{2}+\mathbb{Z}}^{\text {fin }}\right)$. Let

$$
\mathscr{E}^{n, \times}:=\mathscr{E}^{n} / \mathscr{E}^{n, 0},
$$

where $\mathscr{E}^{n, 0}$ is the subspace of $\mathscr{E}^{n}$ spanned by $\left\{E_{\lambda} \mid \lambda \in \Lambda_{\mathbb{Z}^{+}}^{+} \backslash \Lambda_{\mathbb{Z}^{\times}}^{+}\right\}$.

Lemma 6.2. The space $\mathscr{E}^{n, 0}$ is bar-invariant. Hence the bar involution on $\mathscr{E}^{n}$ induces a bar involution on $\mathscr{E}^{n, \times}$.

Proof. By (6.1), we see that the space $\mathscr{E} n, 0$ is spanned by $\left\{L_{\lambda} \mid \lambda \in \Lambda_{\mathbb{Z}}^{+} \backslash \Lambda_{\mathbb{Z}^{\times}}^{+}\right\}$, which proves the claim.

For $\lambda \in \Lambda_{\mathbb{Z}^{x}}^{+}$, put

$$
\mathbf{E}_{\lambda}:=\pi\left(E_{\lambda}\right), \quad \mathbf{L}_{\lambda}:=\pi\left(L_{\lambda}\right),
$$

where $\pi: \mathscr{E}^{n} \rightarrow \mathscr{E}^{n, \times}$ is the canonical projection.

Proposition 6.3. The set $\left\{\mathbf{L}_{\lambda} \mid \lambda \in \Lambda_{\mathbb{Z}^{\times}}^{+}\right\}$is the unique basis of $\mathscr{E}^{n, \times}$ such that $\overline{\mathbf{L}_{\lambda}}=$ $\mathbf{L}_{\lambda}$ and $\mathbf{L}_{\lambda} \in \mathbf{E}_{\lambda}+\sum_{\lambda \succeq \mu, \lambda \neq \mu} q^{-1} \mathbb{Z}\left[q^{-1}\right] \mathbf{E}_{\mu}$ for $\lambda \in \Lambda_{\mathbb{Z}^{\times}}^{+}$.

Proof. By Lemma 6.2 and the bar-invariance of $L_{\lambda}$, we immediately have $\overline{\mathbf{L}_{\lambda}}=\mathbf{L}_{\lambda}$ and $\mathbf{L}_{\lambda} \in \mathbf{E}_{\lambda}+\sum_{\lambda \succeq \mu, \lambda \neq \mu} q^{-1} \mathbb{Z}\left[q^{-1}\right] \mathbf{E}_{\mu}$. The uniqueness follows from [Lu, Lemma 24.2.1].

Define $\mathscr{E}_{\mathbb{Z}\left[q, q^{-1}\right]}^{n, \times}$ and $\mathscr{E}_{\mathbb{Z}}^{n, \times}$ as in (6.2), and put $\mathbf{E}_{\lambda}(1)=1 \otimes \mathbf{E}_{\lambda}, \mathbf{L}_{\lambda}(1)=1 \otimes \mathbf{L}_{\lambda} \in \mathscr{E}_{\mathbb{Z}}^{n, \times}$ for $\lambda \in \Lambda_{\mathbb{Z}^{\times}}^{+}$. We can now translate Theorem 5.3 into Brundan's Fock space language for the Kazhdan-Lusztig theory of $\mathfrak{q}(n)$ [Br2]. 
Theorem 6.4. Let $\Psi: K\left(\mathcal{O}_{\frac{1}{2}+\mathbb{Z}}^{\text {fin }}\right) \rightarrow \mathscr{E}_{\mathbb{Z}}^{\text {n, }} \times$ be the $\mathbb{Z}$-linear isomorphism defined by

$$
[E(\lambda)] \longmapsto \mathbf{E}_{\lambda^{\sharp}}(1) \quad\left(\lambda \in \Lambda_{\frac{1}{2}+\mathbb{Z}}^{+}\right) .
$$

Then $\Psi([L(\lambda)])=\mathbf{L}_{\lambda \sharp}(1)$, for all $\lambda \in \Lambda_{\frac{1}{2}+\mathbb{Z}}^{+}$.

Proof. For $\lambda \in \Lambda_{\frac{1}{2}+\mathbb{Z}}^{+}$, let $\mathbf{L}_{\lambda^{\sharp}}^{\prime}=\Psi([L(\lambda)])$. By (4.7), we have $\mathbf{E}_{\lambda^{\sharp}}(1)=\sum_{\mu} a_{\lambda \mu} \mathbf{L}_{\mu^{\sharp}}^{\prime}$. On the other hand, by (6.1) and Theorem 6.1] we have $\mathbf{E}_{\lambda^{\sharp}}(1)=\sum_{\lambda^{\sharp} \succeq \mu^{\sharp}} a_{\lambda^{\sharp} \mu^{\sharp}} \mathbf{L}_{\mu^{\sharp}}(1)$. Finally by Theorem 5.3, we get

$$
\sum_{\lambda^{\sharp} \succeq \mu^{\sharp}} a_{\lambda^{\sharp} \mu^{\sharp}} \mathbf{L}_{\mu^{\sharp}}(1)=\sum_{\lambda^{\sharp} \succeq \mu^{\sharp}} a_{\lambda^{\sharp} \mu^{\sharp}} \mathbf{L}_{\mu^{\sharp}}^{\prime} .
$$

Since there are only finitely many $\mu$ 's such that $\lambda \succcurlyeq \mu$, we conclude by Corollary 3.4 and induction that $\mathbf{L}_{\mu^{\sharp}}(1)=\mathbf{L}_{\mu^{\sharp}}^{\prime}$ for all $\mu \in \Lambda_{\frac{1}{2}+\mathbb{Z}^{+}}^{+}$. This completes the proof.

Remark 6.5. Theorems 5.3 and 6.4 suggest a connection between the categories $\mathcal{O}_{\frac{1}{2}+\mathbb{Z}}^{\text {in }}$ and $\mathcal{O}_{\mathbb{Z}}^{\text {fin }}$.

For $\lambda \in \Lambda$, we denote by $\lambda^{+}$the unique weight in $\Lambda^{+}$which is $W$-conjugate to $\lambda$. Let $\lambda=\sum_{i=1}^{n} \lambda_{i} \varepsilon_{i} \in \Lambda$ be given. For $1 \leq i<j \leq n$ with $\lambda_{i}+\lambda_{j}=0$, define

$$
\mathrm{R}_{i, j}(\lambda):=\lambda+a\left(\varepsilon_{i}-\varepsilon_{j}\right),
$$

where $a$ is the smallest positive integer such that $\lambda+a\left(\varepsilon_{i}-\varepsilon_{j}\right)$ and all $\mathrm{R}_{k, l}(\lambda)+a\left(\varepsilon_{i}-\varepsilon_{j}\right)$ for $1 \leq k<i<j<l \leq n$ with $\lambda_{k}+\lambda_{l}=0$ are $W$-conjugate to weights in $\Lambda^{+}$.

Let $\lambda \in \Lambda_{\mathbb{Z}^{\times}}^{+}$with the degree of atypicality $r$. Let $1 \leq i_{1}<\ldots<i_{r}<j_{r}<\ldots<j_{1} \leq$ $n$ such that $\lambda_{i_{s}}+\lambda_{j_{s}}=0$ for $1 \leq s \leq r$. Note that $\lambda_{i_{1}}>\cdots>\lambda_{i_{r}}>0>\lambda_{j_{r}}>\cdots>\lambda_{j_{1}}$. Following [Br1, Section 3-f] we define

$$
\begin{aligned}
& \mathrm{R}_{\theta}(\lambda):=\left(\mathrm{R}_{i_{1}, j_{1}}^{\theta_{1}} \circ \mathrm{R}_{i_{2}, j_{2}}^{\theta_{2}} \circ \cdots \circ \mathrm{R}_{i_{r}, j_{r}}^{\theta_{r}}(\lambda)\right)^{+}, \\
& \mathrm{R}_{\theta}^{\prime}(\lambda):=\left(\mathrm{R}_{i_{r}, j_{r}}^{\theta_{r}} \circ \mathrm{R}_{i_{r-1}, j_{r-1}}^{\theta_{r-1}} \circ \cdots \circ \mathrm{R}_{i_{1}, j_{1}}^{\theta_{1}}(\lambda)\right)^{+},
\end{aligned}
$$

for $\theta=\left(\theta_{1}, \ldots, \theta_{r}\right) \in \mathbb{Z}_{+}^{r}$. We put $|\theta|=\sum_{i=1} \theta_{i}$ for $\theta=\left(\theta_{1}, \ldots, \theta_{r}\right) \in \mathbb{Z}_{+}^{r}$.

Theorem 6.6. For $\lambda \in \Lambda_{\frac{1}{2}+\mathbb{Z}}^{+}$with the degree of atypicality $r$, we have

(1) $[E(\lambda)]=\sum_{\mu}[L(\mu)]$, where the sum is over all $\mu \in \Lambda_{\frac{1}{2}+\mathbb{Z}}^{+}$such that $\lambda=\mathrm{R}_{\theta}(\mu)$ for some unique $\theta \in\{0,1\}^{r}$,

(2) $[L(\lambda)]=\sum_{\mu, \theta}(-1)^{|\theta|}[E(\mu)]$, where the sum is over all $\mu \in \Lambda_{\frac{1}{2}+\mathbb{Z}}^{+}$and $\theta \in \mathbb{Z}_{+}^{r}$ such that $\lambda=\mathrm{R}_{\theta}^{\prime}(\mu)$.

Proof. (1) By [Br2, Theorem 3.36], we have for $\alpha, \beta \in \Lambda_{\mathbb{Z}^{\times}}^{+}$

$$
a_{\alpha \beta}= \begin{cases}1, & \text { if } \alpha=\mathrm{R}_{\theta}(\beta) \text { for some } \theta \in\{0,1\}^{r} \\ 0, & \text { otherwise }\end{cases}
$$


where $r$ is the degree of atypicality of $\alpha$. Note that $a_{\alpha \beta}$ is non-zero only if $\alpha \succeq \beta$ and $\alpha, \beta \in \Lambda_{\mathbb{Z}^{\times}}^{+}(p)$ for some $p$. It is easy to see that $\lambda=\mathrm{R}_{\theta}(\mu)$ if and only if $\lambda^{\sharp}=\mathrm{R}_{\theta}\left(\mu^{\sharp}\right)$ for $\lambda, \mu \in \Lambda_{\frac{1}{2}+\mathbb{Z}^{+}}^{+}$. Hence the formula follows from Theorem 6.4

(2) For $\alpha, \beta \in \Lambda_{\mathbb{Z}^{\times}}^{+}$, let

$$
b_{\alpha \beta}=\sum_{\theta}(-1)^{|\theta|}
$$

where the sum is over all $\theta \in \mathbb{Z}_{+}^{r}$ such that $\alpha=\mathrm{R}_{\theta}^{\prime}(\beta)$, and $r$ is the degree of atypicality of $\alpha$ and $\beta$. Note that $b_{\alpha \beta}$ is non-zero only if $\alpha, \beta \in \Lambda_{\mathbb{Z}^{\times}}^{+}(p)$ for some $p$.

Recall that for $f, g \in \mathbb{Z}_{+}^{p \mid q}$, one can define $a_{f g}$ and $b_{f g}$ as in (6.4) and (6.5), where $\mathrm{R}_{\theta}(g)$ and $\mathrm{R}_{\theta}^{\prime}(g)$ are given in [Br1, Section 3-f]. Since $\mathbf{X}_{\theta}\left(\gamma^{b}\right)=\mathbf{X}_{\theta}(\gamma)^{b}$ for $\gamma \in \Lambda_{\mathbb{Z}^{\times}}^{+}(p)$ and $\theta \in \mathbb{Z}_{+}^{r}$ where $\mathrm{X}=\mathrm{R}$ or $\mathrm{R}^{\prime}$, we have by Lemma 3.5 $\alpha=\mathrm{X}_{\theta}(\beta)$ in $\Lambda_{\mathbb{Z}^{\times}}^{+}$if and only if $\alpha^{b}=\mathrm{X}_{\theta}\left(\beta^{b}\right)$ in $\mathbb{Z}_{+}^{p \mid q}$ for $\alpha, \beta \in \Lambda_{\mathbb{Z}^{\times}}^{+}(p)$. This implies that

$$
a_{\alpha \beta}=a_{\alpha^{b} \beta^{b}}, \quad b_{\alpha \beta}=b_{\alpha^{b} \beta^{b}} .
$$

By Proposition 3.6. we also have $\mathrm{R}_{\theta}^{\prime}(\beta) \succeq \beta$ since $\mathrm{R}_{\theta}^{\prime}(\beta)^{b}=\mathrm{R}_{\theta}^{\prime}\left(\beta^{\mathrm{b}}\right) \succeq_{a} \beta^{\mathrm{b}}$ for any $\theta \in \mathbb{Z}_{+}^{r}$ [Br1, Lemma 2.5]. Hence, $b_{\alpha \beta}$ is non-zero only if $\alpha \succeq \beta$.

For $\nu, \mu \in \Lambda_{\frac{1}{2}+\mathbb{Z}}^{+}, b_{\nu \mu}$ is defined in the same way as in (6.5). It is clear that $b_{\nu \mu}=b_{\nu^{\sharp} \mu^{\sharp}}$ since $\nu=\mathrm{R}_{\theta}^{\prime}(\mu)$ if and only if $\nu^{\sharp}=\mathrm{R}_{\theta}^{\prime}\left(\mu^{\sharp}\right)$. Now, for $\lambda, \mu \in \Lambda_{\frac{1}{2}+\mathbb{Z}}^{+}$

$$
\begin{aligned}
\sum_{\nu} a_{\lambda \nu} b_{\nu \mu} & =\sum_{\nu} a_{\lambda^{\sharp} \nu^{\sharp}} b_{\nu^{\sharp} \mu^{\sharp}} & & \text { by Theorem 5.3 } \\
& =\sum_{\lambda^{\sharp} \succeq \nu^{\sharp} \succeq \mu^{\sharp}} a_{\lambda^{\sharp} \nu^{\sharp}} b_{\nu^{\sharp} \mu^{\sharp}} & & \text { by (6.4) }
\end{aligned}
$$

where $\nu \in \Lambda_{\frac{1}{2}+\mathbb{Z}^{+}}^{+}$. The sum in (6.7) is non-zero only when $\lambda^{\sharp}, \mu^{\sharp} \in \Lambda_{\mathbb{Z}^{\times}}^{+}(p)$ for some $p$, and the sum is over $\nu^{\sharp} \in \Lambda_{\mathbb{Z}^{\times}}^{+}(p)$ with $\lambda^{\sharp} \succeq \nu^{\sharp} \succeq \mu^{\sharp}$. In this case, we have

$$
\begin{aligned}
\sum_{\lambda^{\sharp} \succeq \nu^{\sharp} \succeq \mu^{\sharp}} a_{\lambda^{\sharp} \nu^{\sharp}} b_{\nu^{\sharp} \mu^{\sharp}} & =\sum_{\lambda^{\natural} \succeq_{a} \nu^{\natural} \succeq_{a} \mu^{\natural}} a_{\lambda^{\natural} \nu^{\natural}} b_{\nu^{\natural} \mu^{\natural}} & & \text { by (6.6) } \\
& =\sum_{\lambda^{\natural} \succeq_{a} h \succeq_{a} \mu^{\natural}} a_{\lambda^{\natural} h} b_{h \mu^{\natural}} & & \text { by Lemma 3.5 Proposition 3.6 } \\
& =\delta_{\lambda^{\natural} \mu^{\natural}} & & \text { by [Br1], Corollary 3.36], }
\end{aligned}
$$

where $\nu \in \Lambda_{\frac{1}{2}+\mathbb{Z}}^{+}$and $h \in \mathbb{Z}_{+}^{p \mid q}$. By (6.7) and (6.8), we have $\sum_{\nu} a_{\lambda \nu} b_{\nu \mu}=\delta_{\lambda \mu}$. We conclude that $[L(\nu)]=\sum_{\mu} b_{\nu \mu}[E(\mu)]$. The proof completes.

Remark 6.7. Let $\lambda \in \Lambda_{\frac{1}{2}+\mathbb{Z}}^{+}$and let $\mathcal{O}_{\frac{1}{2}+\mathbb{Z}, \chi_{\lambda}}^{\text {fin }}$ denote the subcategory of $\mathcal{O}_{\frac{1}{2}+\mathbb{Z}}^{\text {fin }}$ of modules of central character $\chi_{\lambda}$. Let $p$ and $q$ be determined by $\lambda$ as before (see, e.g., (3.4) ) and let $\mathcal{O}_{p \mid q, \chi_{\lambda}}^{\text {fin }}$ denote the subcategory of finite-dimensional $\mathfrak{g l}(p \mid q)$-modules of $\mathfrak{g l}(p \mid q)$-central character $\chi_{\lambda}$, where $\lambda$ here is regarded as a $\rho$-shifted weight of $\mathfrak{g l}(p \mid q)$. Theorem 6.6, 
together with [Br1, Corollary 3.36], seems to indicate a connection between $\mathcal{O}_{\frac{1}{2}+\mathbb{Z}, \chi_{\lambda}}^{\text {fin }}$ and $\mathcal{O}_{p \mid q, \chi_{\lambda}}^{\text {fin }}$.

\section{KaC-WAKimoto TYPE CharaCter Formulas}

In this section, we derive a closed-form character formula for special classes of finitedimensional irreducible modules, which are similar to Kac-Wakimoto formula for classical Lie superalgebras [KW] (see also [CK, CHR, GK, SZ1]). The results in this section are motivated by [SZ1].

Let $\lambda=\sum_{i=1}^{n} \lambda_{i} \varepsilon_{i} \in \Lambda_{\frac{1}{2}+\mathbb{Z}}^{+}$with the degree of atypicality $r>0$. Let $1 \leq i_{1}<\ldots<$ $i_{r}<j_{r}<\ldots<j_{1} \leq n$ be unique indices such that $\lambda_{i_{s}}+\lambda_{j_{s}}=0$ for $1 \leq s \leq r$. We put $S_{\lambda}=\left\{\beta_{s}:=\varepsilon_{i_{s}}-\varepsilon_{j_{s}} \mid 1 \leq s \leq r\right\} \subset \Phi^{+}$, the set of positive roots atypical to $\lambda$, and define $\lambda^{\Uparrow}$ to be the weight obtained from $\lambda$ by replacing $\lambda_{i_{s}}$ with $\lambda_{i_{1}}$ and replacing $\lambda_{j_{s}}$ with $\lambda_{j_{1}}$ for $2 \leq s \leq n$. Let $\mu, \nu \in \Lambda_{\frac{1}{2}+\mathbb{Z}}$ such that $S_{\mu}=S_{\nu}=S_{\lambda}$. If $\mu \succcurlyeq \nu$ with $\mu=\nu+\sum_{s=1}^{r} c_{s} \beta_{s}$ for some $c_{s} \in \mathbb{Z}_{+}$, then we put $|\mu-\nu|=\sum_{s=1}^{r} c_{s}$.

Following [SZ1, we say that

(1) $\lambda$ is totally connected if for each $1 \leq s \leq r-1$ and $\lambda_{i_{s}}>t>\lambda_{i_{s+1}}$, there exists $1 \leq i \leq n$ such that $\left|\lambda_{i}\right|=t$

(2) $\lambda$ is totally disconnected if for each $1 \leq s \leq r-1$, there exists $\lambda_{i_{s}}>t>\lambda_{i_{s+1}}$ such that $\left|\lambda_{i}\right| \neq t$ for any $1 \leq i \leq n$.

Then we have the following Kac-Wakimoto type character formulas for totally connected and disconnected weights.

Theorem 7.1. Let $\lambda \in \Lambda_{\frac{1}{2}+\mathbb{Z}}^{+}$with the degree of atypicality $r$.

(1) If $\lambda$ is totally connected, then we have

$$
\operatorname{ch} L(\lambda)=\frac{(-1)^{|\lambda \Uparrow-\lambda|} 2^{\lceil n / 2\rceil}}{r ! D} \sum_{w \in W}(-1)^{\ell(w)} w\left(\frac{e^{\lambda^{\lambda}}}{\prod_{\beta \in S_{\lambda}}\left(1+e^{-\beta}\right)}\right) .
$$

(2) If $\lambda$ is totally disconnected, then we have

$$
\operatorname{ch} L(\lambda)=\frac{2^{\lceil n / 2\rceil}}{D} \sum_{w \in W}(-1)^{\ell(w)} w\left(\frac{e^{\lambda}}{\prod_{\beta \in S_{\lambda}}\left(1+e^{-\beta}\right)}\right) .
$$

Proof. (1) Let $\lambda=\sum_{i=1}^{n} \lambda_{i} \varepsilon_{i} \in \Lambda_{\frac{1}{2}+\mathbb{Z}}^{+}$be a totally connected weight. Let $1 \leq i_{1}<$ $\ldots<i_{r}<j_{r}<\ldots<j_{1} \leq n$ be such that $\lambda_{i_{s}}+\lambda_{j_{s}}=0$ for $1 \leq s \leq r$, and $S_{\lambda}=\left\{\beta_{s}=\varepsilon_{i_{s}}-\varepsilon_{j_{s}} \mid 1 \leq s \leq r\right\}$.

Let $\operatorname{KW}(\lambda)$ denote the right-hand side of the equation in (1). For $\mu \in \Lambda_{\frac{1}{2}+\mathbb{Z}}$, put $\sigma(\mu)=2^{\lceil n / 2\rceil} D^{-1} \sum_{w \in W}(-1)^{\ell(w)} w\left(e^{\mu}\right)$, which is equal to $\operatorname{ch} E(\mu)$ by (4.6) when $\mu \in$ 
$\Lambda_{\frac{1}{2}+\mathbb{Z}^{+}}^{+}$Let

$$
\begin{aligned}
X & =\lambda^{\Uparrow}-\sum_{s=1}^{r} \mathbb{Z}_{+} \beta_{s}, \\
X_{1} & =\left\{\lambda^{\Uparrow}-\sum_{s=1}^{r} c_{s} \beta_{s} \in X \mid c_{s} \leq \lambda_{i_{1}}-\frac{1}{2} \text { for all } 1 \leq s \leq r\right\}, \\
X_{2} & =X \backslash X_{1} .
\end{aligned}
$$

Then we have

$$
\mathrm{KW}(\lambda)=\mathrm{KW}_{1}(\lambda)+\mathrm{KW}_{2}(\lambda)
$$

where for $i=1,2$

$$
\mathrm{KW}_{i}(\lambda)=\frac{(-1)^{|\lambda \Uparrow-\lambda|}}{r !} \sum_{\mu \in X_{i}}(-1)^{|\lambda \Uparrow-\mu|} \sigma(\mu)
$$

Suppose that $\lambda^{\sharp} \in \Lambda_{\mathbb{Z}^{\times}}^{+}(p)$ for some $p$. Put $q=n-p$. Then we may identify $\lambda$ with a dominant integral weight $\lambda^{\natural} \in \mathbb{Z}^{p \mid q}$ for $\mathfrak{g l}(p \mid q)$ with respect to the standard Borel subalgebra (cf. [Br1, SZ1]). Note that

$$
\mathrm{KW}_{1}(\lambda)=\sum_{\mu \in \Lambda_{\frac{1}{2}+\mathbb{Z}}^{+}} k_{\lambda \mu} \sigma(\mu),
$$

for some $k_{\lambda \mu} \in \mathbb{Q}$, and $\mu^{\sharp} \in \Lambda_{\mathbb{Z}^{\times}}^{+}(p)$ for $\mu$ such that $k_{\lambda \mu} \neq 0$.

Now, we use the Kac-Wakimoto type character formula for the irreducible $\mathfrak{g l}(p \mid q)$ module with highest weight $\lambda^{\natural}$ [SZ1, Corollary 4.13]. Indeed, we can check without difficulty that the coefficient $k_{\lambda \mu}$ in (7.2) coincides with the coefficient of the character of Kac module with highest weight $\mu^{\natural}$ in the Kac-Wakimoto type formula associated to $\lambda^{\natural}$ (see the right-hand side of (4.46) in [SZ1]). Therefore, by [Br1, Corollary 3.39(ii) and (4.40)] and Theorem 6.6(2), we conclude that

$$
\operatorname{ch} L(\lambda)=\mathrm{KW}_{1}(\lambda)
$$

Next, for $\mu=\sum_{i=1}^{n} \mu_{i} \varepsilon_{i} \in X_{2}$, define $\mu^{\Uparrow-}$ to be the weight obtained from $\mu$ by replacing all the negative (resp. positive) $\mu_{i}$ for $1 \leq i \leq p$ (resp. $p<i \leq n$ ) with $-\frac{1}{2}$ (resp. $\left.\frac{1}{2}\right)$. Put

$$
\overline{X_{2}}=\left\{\nu \mid \nu=\mu^{\Uparrow-} \text { for some } \mu \in X_{2}\right\},
$$

and

$$
I_{\nu}=\left\{\beta \mid \beta=\varepsilon_{i}-\varepsilon_{j} \in S_{\lambda}, \nu_{i}=-\nu_{j}=-1 / 2\right\},
$$


for $\nu \in \overline{X_{2}}$. Then we have

$$
\begin{aligned}
\mathrm{KW}_{2}(\lambda) & =\frac{(-1)^{|\lambda \Uparrow-\lambda|}}{r !} \sum_{\mu \in X_{2}}(-1)^{|\lambda \Uparrow-\mu|} \sigma(\mu) \\
& =\frac{(-1)^{|\lambda \Uparrow-\lambda|}}{r !} \sum_{\nu \in \bar{X}_{2}}(-1)^{\left|\lambda^{\Uparrow}-\nu\right|} \sum_{\left(k_{\beta}\right)_{\beta \in I_{\nu}} \in \mathbb{Z}_{+}^{r}}(-1)^{\sum k_{\beta}} \sigma\left(\nu-\sum_{\beta \in I_{\nu}} k_{\beta} \beta\right) .
\end{aligned}
$$

For each $\nu=\sum_{i=1}^{n} \nu_{i} \varepsilon_{i} \in \overline{X_{2}}$, we have

$$
\sum_{\left(k_{\beta}\right)_{\beta \in I_{\nu}} \in \mathbb{Z}_{+}^{r}}(-1)^{\sum k_{\beta}} \sigma\left(\nu-\sum_{\beta \in I_{\nu}} k_{\beta} \beta\right)=2^{\lceil n / 2\rceil} D^{-1} \sum_{w \in W}(-1)^{\ell(w)} w\left(\frac{e^{\nu}}{\prod_{\beta \in I_{\nu}}\left(1+e^{-\beta}\right)}\right) .
$$

If we put $x_{i}=e^{\varepsilon_{i}}$ for $1 \leq i \leq n$, then

$$
\frac{e^{\nu}}{\prod_{\beta \in I_{\nu}}\left(1+e^{-\beta}\right)}=\frac{x_{1}^{\nu_{1}} \cdots x_{n}^{\nu_{n}}}{\prod_{\beta=\varepsilon_{i}-\varepsilon_{j} \in I_{\nu}}\left(1+x_{i}^{-1} x_{j}\right)}=\frac{x_{1}^{\nu_{1}^{\prime}} \cdots x_{n}^{\nu_{n}^{\prime}}}{\prod_{\beta=\varepsilon_{i}-\varepsilon_{j} \in I_{\nu}}\left(x_{i}+x_{j}\right)},
$$

where $\nu^{\prime}=\sum_{i=1}^{n} \nu_{i}^{\prime} \varepsilon_{i}=\nu+\sum_{\beta=\varepsilon_{i}-\varepsilon_{j} \in I_{\nu}} \varepsilon_{i}$. Since $\nu_{i}^{\prime}=\nu_{j}^{\prime}=1 / 2$ for $\beta=\varepsilon_{i}-\varepsilon_{j} \in I_{\nu}$, the product form in (7.5) is invariant under the transposition $(i, j) \in W$, which in particular implies that the alternating sum (17.4) is zero, and accordingly

$$
\mathrm{KW}_{2}(\lambda)=0 \text {. }
$$

Therefore, the formula follows from (7.1), (77.3), and (7.6).

(2) The proof is almost the same as in (1), where we replace $\lambda^{\Uparrow}$ with $\lambda$, and apply [SZ1, Corollary 4.15]. We leave the details to the reader.

\section{REFERENCES}

[BL] I.N. Bernstein and D.A. Leites, A formula for the characters of the irreducible finitedimensional representations of Lie superalgebras of series $\mathfrak{g l}$ and $\mathfrak{s l}$ (Russian), C. R. Acad. Bulgare Sci. 33 (1980), 1049-1051.

[Br1] J. Brundan, Kazhdan-Lusztig polynomials and character formulae for the Lie superalgebra $\mathfrak{g l}(m \mid n)$, J. Amer. Math. Soc. 16 (2003), 185-231.

[Br2] J. Brundan, Kazhdan-Lusztig polynomials and character formulae for the Lie superalgebra $\mathfrak{q}(n)$, Adv. Math. 182 (2004), 28-77.

[CK] S.-J. Cheng and J.-H. Kwon, Kac-Wakimoto character formula for ortho-symplectic Lie superalgebras, preprint (2014), arXiv:1406.6739.

[CL] S.-J. Cheng and N. Lam, Irreducible characters of general linear superalgebra and super duality, Commun. Math. Phys. 280 (2010), 645-672.

[CLW] S.-J. Cheng, N. Lam, and W. Wang, Super duality and irreducible characters of orthosymplectic Lie superalgebras, Invent. Math. 183 (2011), 189-224.

[CW] S.-J. Cheng and W. Wang, Dualities and Representations of Lie Superalgebras. Graduate Studies in Mathematics 144. American Mathematical Society, Providence, RI, 2012. 
[CHR] M. Chmutov, C. Hoyt. and S. Reif, Kac-Wakimoto character formula for the general linear Lie superalgebra, preprint (2013), arXiv:1310.3798.

[Ger] J. Germoni, Indecomposable representations of $\operatorname{osp}(3,2), D(2,1 ; \alpha)$ and $G(3)$, Colloquium on Homology and Representation Theory (Spanish) (Vaquerias, 1998), Bol. Acad. Nac. Cienc. (Cordoba) 65 (2000), 147-163.

[GK] M. Gorelik and V. Kac, Characters of (relatively) integrable modules over affine Lie superalgebras, preprint (2014), arXiv:1406.6860.

[GS] C. Gruson and V. Serganova, Cohomology of generalized supergrassmannians and character formulae for basic classical Lie superalgebras, Proc. Lond. Math. Soc. 101 (2010), 852-892.

[JMO] N. Jing, K. Misra, and M. Okado, q-Wedge Modules for Quantized Enveloping Algebra of Classical Type, J. Algebra 230 (2000), 518-539.

[KV] A. Knapp and D. Vogan, Cohomological induction and unitary representations, Princeton Mathematical Series, 45. Princeton University Press, Princeton, NJ, 1995

[KW] V. Kac and M. Wakimoto, Representations of affine superalgebras and mock theta functions, preprint (2013), arXiv:1308.1261.

[Lu] G. Lusztig, Introduction to quantum groups, Progress in Math. 110, Birkhäuser, 1993.

[Mar] L. Martirosyan, The representation theory of the exceptional Lie superalgebras $F(4)$ and $G(3)$, J. Algebra 419 (2014), 167-222.

[Pe] I. Penkov, Characters of typical irreducible finite-dimensional $\mathfrak{q}(n)$-modules, Funct. Anal. App. 20 (1986), 30-37.

[PS1] I. Penkov and V. Serganova, Characters of Finite-Dimensional Irreducible $\mathfrak{q}(n)$-Modules, Lett. Math. Phys. 40 (1997), 147-158.

[PS2] I. Penkov and V. Serganova, Characters of irreducible G-modules and cohomology of $G / P$ for the supergroup $G=Q(N)$, J. Math. Sci., 84 (1997), 1382-1412.

[San] J. Santos, Foncteurs de Zuckerman pour les superalgébres de Lie, J. Lie Theory 9 (1999), 69-112.

[Ser] V. Serganova, Characters of irreducible representations of simple Lie superalgebras, Doc. Math., Extra Volume ICM II (1998), 583-593.

[Sv1] A. Sergeev, The tensor algebra of the identity representation as a module over the Lie superalgebras $g l(n, m)$ and $Q(n)$, Math. USSR Sbornik 51 (1985), 419-427.

[Sv2] A. Sergeev, The centre of enveloping algebra for Lie superalgebra $Q(n, \mathbb{C})$, Lett. Math. Phys. 7 (1983), 177-179.

[SZ1] Y. Su and R. B. Zhang, Character and dimension formulae for general linear superalgebra, Adv. Math. 211 (2007), 1-33.

[SZ2] Y. Su and R.B. Zhang, Generalized Jantzen filtration of Lie superalgebras II: the exceptional cases, preprint (2013), arXiv:1303.4797.

[SZ3] Y. Su and R.B. Zhang, Character and dimension formulae for queer Lie superalgebra, Comm. Math. Phys. 333 (2015), 1465-1481.

[vdJ] J. Van der Jeugt, Character formulae for Lie superalgebra C(n), Comm. Algebra 19 (1991), 199-222.

Institute of Mathematics, Academia Sinica, Taipei, Taiwan 10617

E-mail address: chengsj@math.sinica.edu.tw

Department of Mathematics, Sungkyunkwan University 2066 Seobu-ro, Jangan-gu, SuWON, KOREA

E-mail address: jaehoonkw@skku.edu 